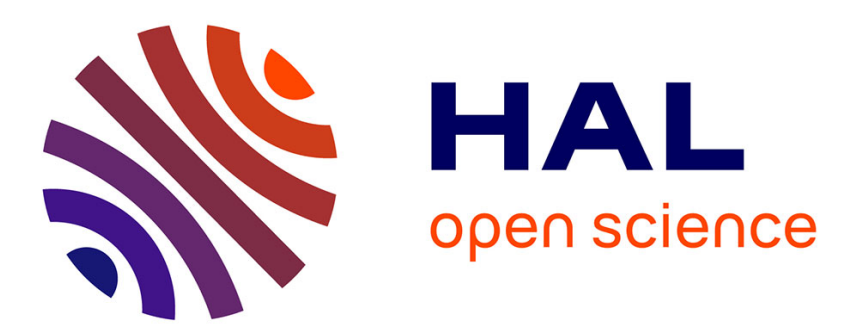

\title{
Identification of crystal plasticity parameters using DIC measurements and weighted FEMU
}

Adrien Guery, François Hild, Félix Latourte, Stéphane Roux

\section{To cite this version:}

Adrien Guery, François Hild, Félix Latourte, Stéphane Roux. Identification of crystal plasticity parameters using DIC measurements and weighted FEMU. Mechanics of Materials, 2016, 100, pp.55 71. 10.1016/j.mechmat.2016.06.007 . hal-01383934

\section{HAL Id: hal-01383934 https://hal.science/hal-01383934}

Submitted on 19 Oct 2016

HAL is a multi-disciplinary open access archive for the deposit and dissemination of scientific research documents, whether they are published or not. The documents may come from teaching and research institutions in France or abroad, or from public or private research centers.
L'archive ouverte pluridisciplinaire HAL, est destinée au dépôt et à la diffusion de documents scientifiques de niveau recherche, publiés ou non, émanant des établissements d'enseignement et de recherche français ou étrangers, des laboratoires publics ou privés. 


\title{
Identification of crystal plasticity parameters using DIC measurements and weighted FEMU
}

\author{
Adrien Guery ${ }^{\mathrm{a}, \mathrm{b}}$, François Hild ${ }^{\mathrm{a}}$, Félix Latourte ${ }^{\mathrm{b}}$, Stéphane Roux ${ }^{\mathrm{a}}$ \\ ${ }^{a}$ LMT-Cachan (ENS Cachan/CNRS/University Paris Saclay) \\ 61 avenue du Président Wilson, F-94235 Cachan (FRANCE) \\ ${ }^{b} E D F R \mathcal{G} D$, Site des Renardières, avenue des Renardières - Ecuelles, F-77818 Moret-sur-Loing (FRANCE)
}

\begin{abstract}
An inverse method for the identification of a set of crystal plasticity parameters is introduced and applied to in situ tensile tests on steel polycrystals. Various mean grain sizes are obtained with different conditioning of AISI 316LN austenitic stainless steel. Identification is based on a weighted Finite Element Model Updating (FEMU) using both displacement fields at the microscale and macroscopic load levels. The values of the identified parameters depend on the factor weighing the contributions of the microscopic and macroscopic quantities. On the one hand, surface displacement fields are measured by Digital Image Correlation (DIC). On the other hand, they are simulated by resorting to Finite Element calculations with a local crystal plasticity law (i.e., Méric-Cailletaud's model) and a 2D simulation of the microstructure. The parameters associated with isotropic hardening are calibrated for the different mean grain sizes. The benefits of considering full-field measurements are manifest for instance in the excellent Hall-Petch trend captured at the microstructural scale. The identification procedure is also applied to the estimation of hardening parameters describing the interaction between slip systems.

Keywords: Crystal plasticity, Identification, Digital Image Correlation
\end{abstract}

\section{Introduction}

Crystal plasticity models allow the mechanical behavior of heterogeneous materials to be finely described at the scale of their microstructure. Numerical simulations using such models give access to local stress and strain fields and more specifically the localizations of these fields associated with microstructural features such as grain boundaries. These calculations are gener- 
ally conducted by using finite element methods [1] or Fast-Fourier Transform techniques [2]. The field localizations are of major importance when the degradation mechanisms occurring at the grain scale(e.g., such as the phenomena of fatigue crack initiation and propagation, intergranular fracture or stress corrosion cracking) are investigated. Since the constitutive equations of crystal plasticity models are written at the single-crystal level, their relevance to describe the polycrystal behavior requires experimental validations. This step deals with the challenging scale transition between single-crystal and polycrystals, which involves the mechanical role of grain boundaries on plasticity.

In this context, full-field measurements provide spatially dense experimental information that can be used to validate both constitutive laws and microstructural calculations. For instance, a direct comparison between DIC measurements and crystal plasticity calculations in polycrystals for the austenitic stainless steel A316LN has been addressed in Ref. [3]. A good agreement in terms of kinematic fields and major slip system activity between experimental measurements and computations has been found. However, some differences remain in the local fields that may be minimized by optimizing the model parameters, which is the topic of the present paper.

Various studies have successfully exploited full-field measurements for the parameter identification of macroscopic constitutive laws [4-7]. Such identification approaches using full-field measurements are specifically relevant when heterogeneous mechanical fields $[4,6]$ or heterogeneous material properties $[6,8]$ are considered. It is worth noting that the measurements may be performed in some cases using images acquired from optical microscopy in order to take into account the effects of the material microstructure on the experimental fields [5].

At the microstructural scale, the use of Scanning Electron Microscope (SEM) imaging provides intragranular DIC measurements with high spatial resolution on the surface of polycrystals that make possible the coupling with crystal plasticity calculations [3, 9-12]. Such high resolution measurements allow not only the microstructure modeling involved in polycrystal calculations to be validated but also the intragranular variations of the simulated fields. However, only few studies have exploited this experimental information for the identification of crystal plasticity parameters $[9,13]$ or to address the quantitative agreement between polycrystalline models and experiments $[3,10,14-16]$. 
In this study, it is first proposed to discuss the choices made to simulate experimental tensile tests on polycrystals. The second part is devoted to the identification procedure based on both microstructural displacement fields and the material homogenized behavior. Because the tensile tests considered herein have no unloading phases, only the isotropic hardening part of the considered crystal plasticity model will be studied. Last, the identification of the corresponding parameters for the law proposed by Méric et al. [17] and the interaction parameters of slip systems are presented and discussed.

\section{Finite Element model}

This first part presents the assumptions used for modeling the local and averaged responses of polycrystals loaded during an in situ (i.e., inside an SEM) tensile test. The material, the experimental facilities and the displacement fields measurements by DIC have already been introduced in detail in Ref. [3].

\subsection{Simulation of the local response of polycrystals}

The simulation of the local response of polycrystals is performed by finite element calculations using Code_Aster (http://www.code-aster.org/). The choice has been made to work with the phenomenological crystal plasticity law proposed by Méric et al. [17]. Its constitutive equations and the parameters are detailed in AppendixA. The material considered in this study is a 316LN austenitic stainless steel of face centered cubic (FCC) crystal lattice. The constitutive relationships are expressed for each of its twelve octahedral slip systems $s$. While many other crystal constitutive laws are available [1], the choice of the present model has been motivated by its good agreement between numerical slip predictions in polycrystals and experimental measurements [3]. Moreover, this law is numerically efficient when implemented in a finite element code, in terms of Newton iterations and computation time.

An underlying difficulty when simulating the local response of experimental polycrystals is to model their microstructure using the available experimental information. In the present study, the microstructure has been characterized in the so-called Region Of Interest (ROI) of each specimen by Electron Back-Scattered Diffraction (EBSD), which leads to 2D surface fields. The lack of 
knowledge in the bulk of the experimental microstructure may potentially induce a modeling error that needs to be accounted for. In this work, the use of 2D models with different modeling strategies is proposed. Since the crystal plasticity law expects a $3 \mathrm{D}$ integration, an assumption in the out-of-plane direction is required. In particular, two assumptions are compared in the following. The first one, referred to as $2 \mathrm{D}$, consists of approaching the plane stress condition. The 3D strain and stress tensors are expressed as

$$
\boldsymbol{\epsilon}=\left[\begin{array}{ccc}
\epsilon_{x x} & \epsilon_{x y} & 0 \\
\epsilon_{x y} & \epsilon_{y y} & 0 \\
0 & 0 & \epsilon_{z z}
\end{array}\right] \quad \boldsymbol{\sigma}=\left[\begin{array}{ccc}
\sigma_{x x} & \sigma_{x y} & 0 \\
\sigma_{x y} & \sigma_{y y} & 0 \\
0 & 0 & \sigma_{z z}
\end{array}\right]
$$

where $z$ is the normal direction to the image plane and with $\sigma_{z z}$ tending to zero with the Newton iterations $i$ of the numerical solution

$$
\sigma_{z z}^{(i)} \rightarrow 0
$$

This method allows for the use of a 2D mesh, which is chosen as the same employed for the DIC measurement, taking as support the microstructure grain or twin boundaries [3]. An alternative route, designated as quasi-2D, consists of extruding the above mesh perpendicular to the surface with one element through the thickness (with a depth equal to the characteristic length of the 2D mesh). The mesh made of triangular prisms is then cut into 4-noded tetrahedra. For both assumptions, experimentally measured displacements are prescribed with their time history on the edges of the meshes. For the quasi-2D mesh, zero displacements along the normal of the surface are prescribed on the back face. According to the results of previous studies [9, 18], these choices for modeling the microstructure appear the least arbitrary and the most realistic ones when the microstructure is unknown in the bulk.

To compare the two assumptions, finite element calculations are performed, one with the 2D mesh, the other one with the quasi-2D mesh, both with the same boundary conditions and the same set of parameters given in AppendixA. The assumption of small strain levels is adopted herein since the calculations are not conducted farther than a mean strain of $5 \%$. For illustration purposes, one of the studied experimental microstructures, denoted as $D_{50}$, is chosen to perform 
this comparison. Figure 1 shows the displacement fields obtained with the 2D assumption for a macroscopic strain of about $5 \%$. The difference between the two calculations in terms of the

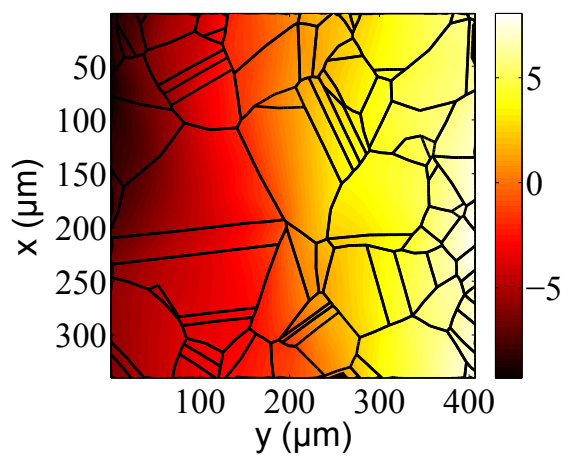

(a)

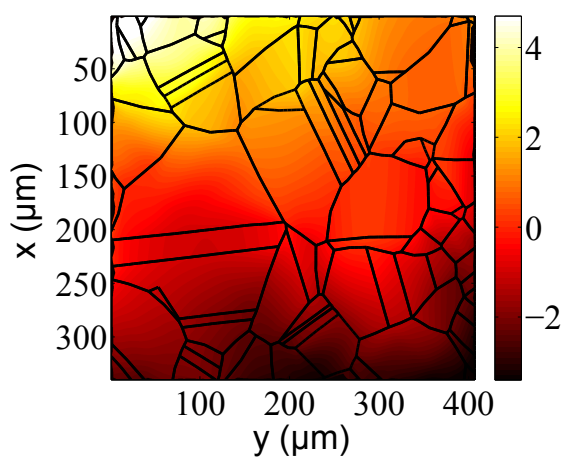

(b)

Figure 1: Displacement fields expressed in micrometers along the horizontal $y$ (a) and the vertical $x$ (b) axes simulated with the $2 \mathrm{D}$ assumption for a macroscopic strain of about $5 \%$

displacement fields obtained on the surface are presented in Figure 2. On these fields and for all the following ones the microstructure boundaries are drawn as solid lines. The displacement difference lies in the $1.5 \mu \mathrm{m}$ range while the dynamic range (i.e., amplitude) of the displacement fields is $17 \mu \mathrm{m}$ at this loading step. Although not negligible, the difference is small and localized in the vicinity of some microstructure boundaries (the standard deviation of the difference is equal to $0.4 \mu \mathrm{m}$ ). As a consequence, the $2 \mathrm{D}$ assumption has been selected for the identification method based on the displacement fields (see Section 3) since it is by far more computationally efficient. For example, the presented calculation takes about $3 \mathrm{~h}$ with the $2 \mathrm{D}$ assumption and about $22 \mathrm{~h}$ with the quasi-2D assumption, both with MPI parallel computations on 8 processors.

As a comparison, the standard measurement uncertainty for this microstructure is equal to $7.1 \mathrm{~nm}$ [3]. It is much lower than the gap between the two simulated fields at a macroscopic strain of $5 \%$. This shows that whatever assumption is made, the modeling error is not negligible and may be reduced thanks to the identification of the crystal plasticity law parameters. 


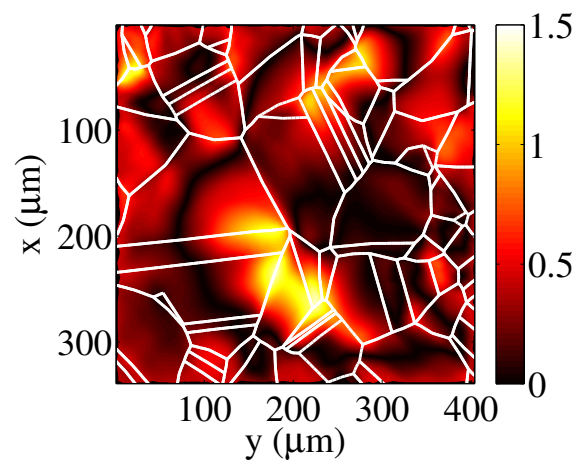

(a)

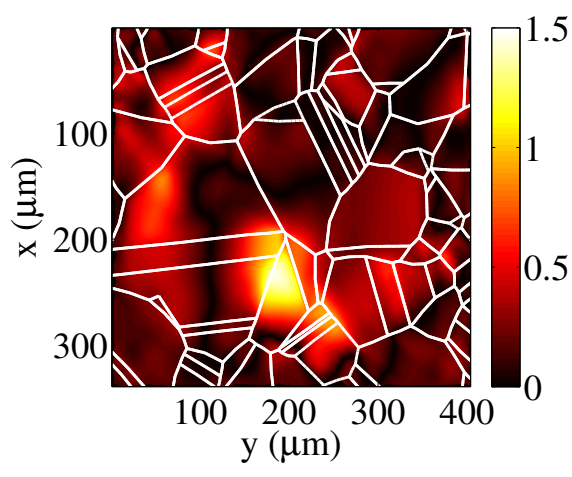

(b)

Figure 2: Absolute difference expressed in micrometers between the displacement fields along the horizontal $y$ (a) and the vertical $x$ (b) axes for a macroscopic strain of about $5 \%$, when simulated with the 2D and quasi-2D assumptions

\subsection{Simulation of the effective behavior}

The Region Of Interest (ROI) for which the DIC measurements are performed encompasses an insufficient number of grains to be considered as a representative volume element. The aim of the measurements is to get a spatially dense kinematic measurement inside grains and close to their boundaries, thereby motivating the selection of a small number of grains in the ROI. Computing the material effective behavior needed in the identification procedure (see Section 3) can therefore not be achieved using a full $2 \mathrm{D}$ field model corresponding to the ROI and an alternative method is proposed in the following.

For specimen $D_{50}$, a sequence of EBSD measurements is conducted and stitched in order to determine the microstructure over the entire width of the specimen (i.e., over $1.9 \mathrm{~mm}$ ), including the area, denoted ROI 1, in which displacement fields are measured by DIC and simulated. This large field microstructure is denoted as ROI 2. Figure 3 shows the full EBSD Inverse Pole Figure (IPF) map obtained in this region over which is drawn the grain boundary map.

The simulation of the in situ tensile test is performed considering the microstructure of ROI 2 , using the law proposed by Méric et al. and the same set of parameters as for the calculation on ROI 1. For that purpose, a 2D finite element mesh is built taking as support the microstructure of ROI 2 with the same characteristic length as the previous ROI 1 mesh. Moreover, the calcu- 


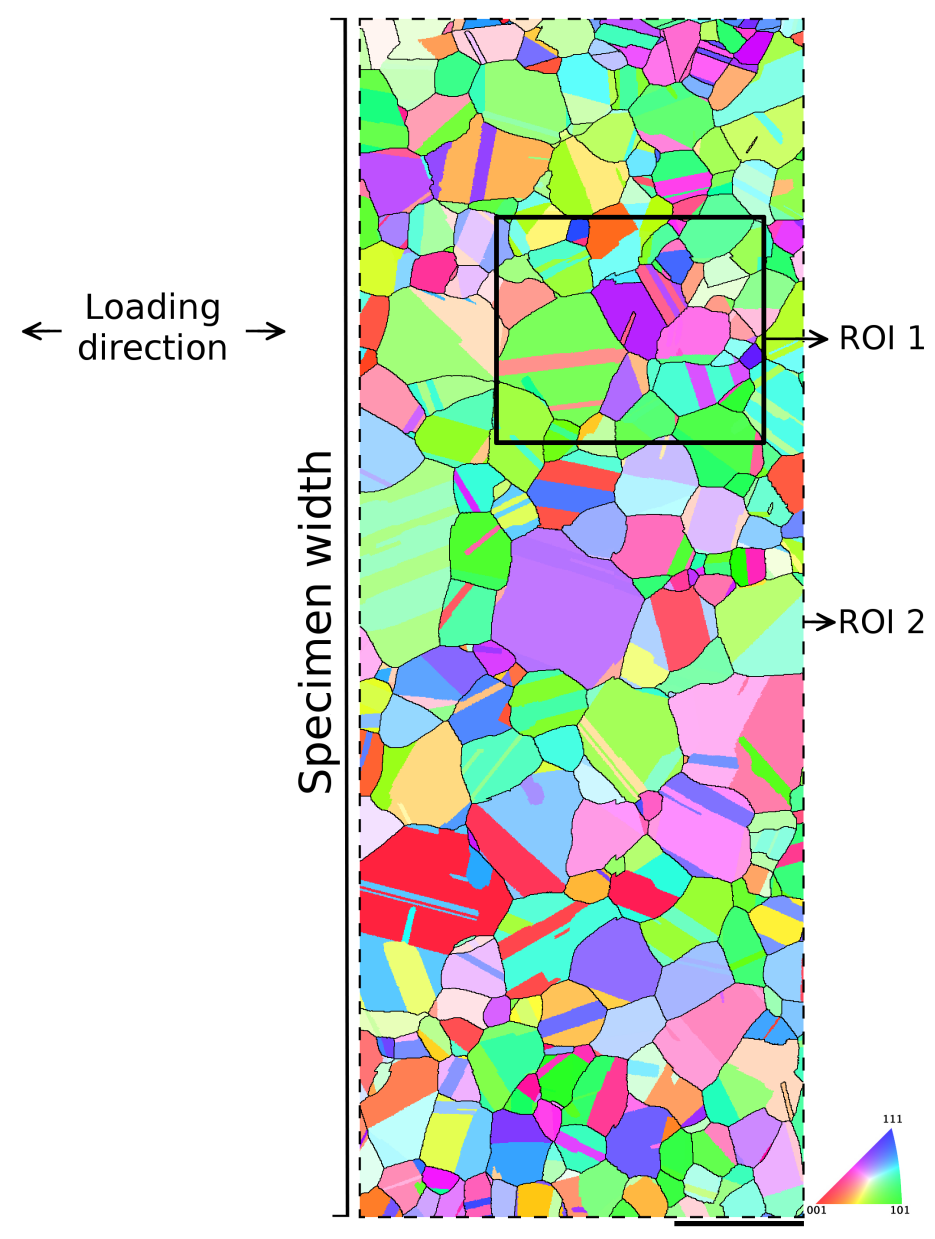

Figure 3: IPF and grain boundary maps of the full width of specimen $D_{50}$. The edges of the regions of interest denoted ROI 1 and ROI 2 are drawn with solid and dashed lines, respectively. The scale bar is $200 \mu \mathrm{m}$ long, and the specimen width (vertical in the figure) is $2 \mathrm{~mm}$

lation is performed with a plane stress assumption. As to boundary conditions, a homogeneous displacement corresponding to the experimental macroscopic strain is applied along the horizontal direction for the vertical edges. The horizontal edges matching with the specimen edges are modeled as traction-free.

The mean values of the obtained stress and strain fields along the loading direction and over the whole surface allow us to compute the stress-strain curve shown in Figure 4. The latter is compared with that obtained from the $2 \mathrm{D}$ calculation previously performed on ROI 1 . Important stress fluctuations are observed on the second curve, which may be the consequence of the low 
number of grains in ROI 1 and of the experimentally measured displacements prescribed on the edges. Despite these fluctuations, the effective behaviors simulated by the two calculations are similar. However, compared with the experimental macroscopic stress-strain curve, the simulated effective behaviors significantly underestimate the stress level.

This difference is unexpected since the three parameters associated with isotropic hardening of the crystal plasticity law used for the two calculations are identified by homogenization from the experimental curve considering a random texture. For that purpose, Berveiller-Zaoui's model is used [19] considering a uniformly-distributed random sampling of 300 crystallographic orientations. Figure 4 shows a very good agreement between the experimental data and the response of the model at convergence. The difference in stress levels between the latter and the macroscopic behavior obtained with the calculation on ROI 2 seems to be related to the plane stress assumption. This gap is reduced by half when the calculation on ROI 1 is performed using the quasi-2D assumption. Consequently, Berveiller-Zaoui's model will be utilized in the following to estimate the macroscopic behavior of the material from a given set of parameters of the crystal plasticity law.

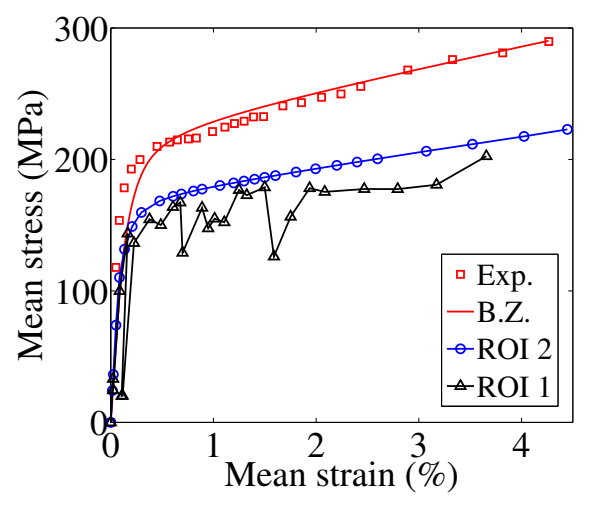

Figure 4: Stress-strain curves corresponding to the mean values of the fields obtained from the 2D calculations on ROI 1 and ROI 2, from the homogenization calculation (B.Z.) and experimental curve (Exp.) 


\section{Weighted FEMU}

\subsection{Identification method}

This part is devoted to the presentation of the inverse method used in this work to identify crystal plasticity parameters. It is based on the minimization of a cost function $\chi_{T}$ that is the combination of two least squares criteria, namely, one dealing with the measured displacement fields at the microstructural scale, denoted by $\chi_{u}$, and the other one with the measured load level at the macroscopic scale, denoted by $\chi_{F}$, such that

$$
\chi_{T}^{2}(\{\boldsymbol{p}\})=\frac{N_{d o f}}{1+N_{d o f}} \chi_{u}^{2}(\boldsymbol{p})+\frac{1}{1+N_{d o f}} \chi_{F}^{2}(\boldsymbol{p})
$$

where $\{\boldsymbol{p}\}$ is the column vector gathering the parameters to identify, and $N_{d o f}$ the number of degrees of freedom of the mesh. This combination allows the (dimensionless) cost function $\chi_{T}$ to have a mathematical expectation equal to unity in absence of model error. Any model error probed via kinematic and static data will induce an increase of $\chi_{T}$. The larger $\chi_{T}$ the higher the model error.

On the one hand, the expression of $\chi_{u}$ reads

$$
\chi_{u}^{2}(\{\boldsymbol{p}\})=\frac{1}{N_{d o f} N_{t}} \sum_{t}\{\boldsymbol{\delta} \boldsymbol{u}\}_{t}^{T}\left[\operatorname{Cov}_{u}\right]^{-1}\{\boldsymbol{\delta} \boldsymbol{u}\}_{t}
$$

with $\{\boldsymbol{\delta} \boldsymbol{u}\}_{t}$ the column vector of the difference evaluated at each degree of freedom between measured displacements $\left\{\boldsymbol{u}_{m}\right\}_{t}$ and simulated ones $\left\{\boldsymbol{u}_{c}(\{\boldsymbol{p}\})\right\}_{t}$ at time step $t . N_{d o f}$ and $N_{t}$, the number of time steps, are introduced to normalize $\chi_{u}^{2}$. In absence of model error, the mathematical expectation of $\chi_{u}$ will be equal to 1 . The covariance matrix $\left[\mathbf{C o v}_{u}\right]$ of the measured kinematic degrees of freedom is used to weight the least squares criterion since it is a known quantity related to the DIC matrix $[M]$ determined at each time step [20], such that

$$
\left[\operatorname{Cov}_{u}\right]=2 \eta_{f}^{2}[M]^{-1}
$$

where $\eta_{f}$ is the standard deviation of noise on SEM images, assumed to be white and Gaussian. On the other hand, $\chi_{F}$ is given by 


$$
\chi_{F}^{2}(\{\boldsymbol{p}\})=\frac{1}{\eta_{F}^{2} N_{t}}\{\boldsymbol{\delta} \boldsymbol{F}\}^{T}\{\boldsymbol{\delta} \boldsymbol{F}\}
$$

where $\{\boldsymbol{\delta} \boldsymbol{F}\}$ is the column vector of the difference evaluated at each time step $t$ between the experimental load $\left\{\boldsymbol{F}_{m}\right\}$ and that obtained by a homogenization calculation $\left\{\boldsymbol{F}_{c}(\{\boldsymbol{p}\})\right\}$ with BerveillerZaoui's model and the current value of $\{\boldsymbol{p}\}$. A normalization by the number of time steps is used to be consistent with the definition of $\chi_{u}^{2}$. Similarly, the standard deviation of the load measurement, denoted by $\eta_{F}$, is introduced to normalize $\chi_{F}$.

All these normalizations imply that $\chi_{u}$ and $\chi_{F}$ are dimensionless. The closer their value to unity, the closer the gap between the simulation and the experiment to measurement resolution, in terms of displacement fields and macroscopic stresses respectively. In practice, the model imperfections imply that the cost functions are strictly greater than one but can be minimized by optimizing the crystal plasticity parameters.

The balance in this identification method between the microstructural $\left(\chi_{u}\right)$ and the macroscopic $\left(\chi_{F}\right)$ scales appeared to give too much weight to the displacement gap. The parameters associated with isotropic hardening of the law proposed by Méric et al. and identified with the presented method led to an unrealistic predicted effective behavior (see Section 4). This result may be the consequence of a limitation of the constitutive law in describing the locally observed hardening, of a modeling error induced by the lack of knowledge in the experimental three-dimensional bulk morphology, or of too noisy boundary conditions extracted from the DIC analysis and that feeds the numerical model. To allow the parameters of a given crystal plasticity law to be optimized according to both microstructural displacement fields and macroscopic stresses, a weight $w$ is introduced to define a global residual

$$
\chi_{T}^{2}(\{\boldsymbol{p}\})=(1-w) \chi_{u}^{2}(\{\boldsymbol{p}\})+w \chi_{F}^{2}(\{\boldsymbol{p}\})
$$

Its value is to be chosen between 0 and 1. This weight has been utilized in a previous study for the identification of Ramberg-Osgood's parameters [7]. In the present case, when $w=0$, the identification is only based on the local displacement fields, while it only takes into account the effective (i.e., macroscopic) behavior when $w=1$. Note that the natural choice of $w$ based 
on the balance between the measurement uncertainties of the two sources of information (i.e., $\left.w=1 /\left(1+N_{d o f}\right)\right)$ is of the order of $10^{-4}$.

The minimization of the cost function $\chi_{T}$ with respect to the parameters $\{\boldsymbol{p}\}$ is performed iteratively via a Newton-Raphson algorithm. At iteration $n$, the parameter corrections $\left\{\boldsymbol{\delta} \boldsymbol{p}^{(n)}\right\}$ are given by

$$
\left((1-w)\left[\boldsymbol{H}_{u}^{(n-1)}\right]+w\left[\boldsymbol{H}_{F}^{(n-1)}\right]\right)\left\{\boldsymbol{\delta} \boldsymbol{p}^{(n)}\right\}=(1-w)\left\{\boldsymbol{h}_{u}^{(n-1)}\right\}+w\left\{\boldsymbol{h}_{F}^{(n-1)}\right\}
$$

with the Hessian matrices expressed as

$$
\begin{gathered}
{\left[\boldsymbol{H}_{u}^{(n-1)}\right]=\frac{1}{2 \eta_{f}^{2} N_{d o f} N_{t}} \sum_{t}\left[\boldsymbol{S}_{u}^{(n-1)}\right]_{t}^{T}[\boldsymbol{M}]\left[\boldsymbol{S}_{u}^{(n-1)}\right]_{t}} \\
{\left[\boldsymbol{H}_{F}^{(n-1)}\right]=\frac{1}{\eta_{F}^{2} N_{t}}\left[\boldsymbol{S}_{F}^{(n-1)}\right]^{T}\left[\boldsymbol{S}_{F}^{(n-1)}\right]}
\end{gathered}
$$

and with

$$
\begin{gathered}
\left\{\boldsymbol{h}_{u}^{(n-1)}\right\}=\frac{1}{2 \eta_{f}^{2} N_{d o f} N_{t}} \sum_{t}\left[\boldsymbol{S}_{u}^{(n-1)}\right]_{t}^{T}[\boldsymbol{M}]\{\boldsymbol{\delta} \boldsymbol{u}\}_{t} \\
\left\{\boldsymbol{h}_{F}^{(n-1)}\right\}=\frac{1}{\eta_{F}^{2} N_{t}}\left[\boldsymbol{S}_{F}^{(n-1)}\right]^{T}\{\boldsymbol{\delta} \boldsymbol{F}\}
\end{gathered}
$$

where $\left[\boldsymbol{S}_{u}^{(n-1)}\right]_{t}$, respectively $\left[\boldsymbol{S}_{F}^{(n-1)}\right]$, is the sensitivity matrix of the displacement fields at the time step $t$, respectively of the macroscopic stress, evaluated from the current value of the parameters $\left\{\boldsymbol{p}^{(n)}\right\}$.

\subsection{Test cases}

The identification method is now studied for two numerical test cases. The first one consists of identifying known reference values of the parameters $r_{0}, q$ and $b$ associated with isotropic hardening. The "experimental" displacement fields, respectively macroscopic stress-strain curve, are obtained from a first finite element calculation, respectively homogenization calculation, performed with the reference values of the parameters. No noise is considered on the reference data in this study and $\left[\mathbf{C o v}_{u}\right]$ is chosen equal to the identity matrix. As a consequence, $\chi_{u}$ and $\chi_{F}$ 
can reach values lower than one. For the finite element calculation, a simplified microstructure made of four grains is considered and the 2D assumption is used. The parameters to identify are initially set with a relative difference of about $20 \%$ from the reference values. The identification is performed with an equal weight given to both cost functions (i.e., $w=1 / 2$ ).

Figure 5 shows the changes of the cost functions $\chi_{u}$ and $\chi_{F}$, of the load, and of the three parameters to be identified simultaneously with the number of iterations of the identification procedure. 20 iterations are required to identify the three parameters with an absolute error less than $10^{-4}$. The isotropic hardening parameter $r_{0}$ is determined first. The effective behavior is also quickly captured as shows the tenth iteration in Figure 5b. The difficulty in this test case is that $q$ and $b$ are to be identified simultaneously while these parameters have a very similar role in the isotropic hardening law. For low values of $b$ the hardening rate becomes linear and controlled only by the $b q$ product (see Equation (A.4)). As soon as $r_{0}$ and the effective behavior are close enough to the reference data (i.e., from iteration 8 on), $q$ and $b$ start to converge to their reference values. If the same exercise is performed with $r_{0}$ and $q$ to be identified and $b$ kept fixed, only 4 iterations are then required to solve the problem with the same final value of $\chi_{u}$ and $\chi_{F}$.

A second test case is conducted to address the errors associated with the 2D modeling hypothesis. A finite element calculation of a 3D polycrystalline aggregate obtained with a Voronoi tessellation is used to generate the "experimental" data. It consists of a tensile loading of a cube composed of 300 grains with randomly-distributed orientations producing an isotropic texture. The normal displacement on each face of the cube is prescribed as uniform (i.e., planar boundary conditions). The loading is prescribed on the bottom and top faces. Then, the reference displacement field is read on one of the lateral face, denoted the "observed" face. The reference stress-strain curve is obtained by averaging the longitudinal strain and stress components over the whole volume. The observed face is then extracted for a $2 \mathrm{D}$ calculation, with the same $2 \mathrm{D}$ mesh and crystallographic orientations. The same boundary conditions are applied, namely, a normal uniform displacement on each edge. Thus, the identification of crystal plasticity parameters is performed by minimizing the gap between the local response of this $2 \mathrm{D}$ calculation and the reference fields. As previously, the calculated stress-strain curve is given at each iteration by the homogenization model and $w=1 / 2$. 


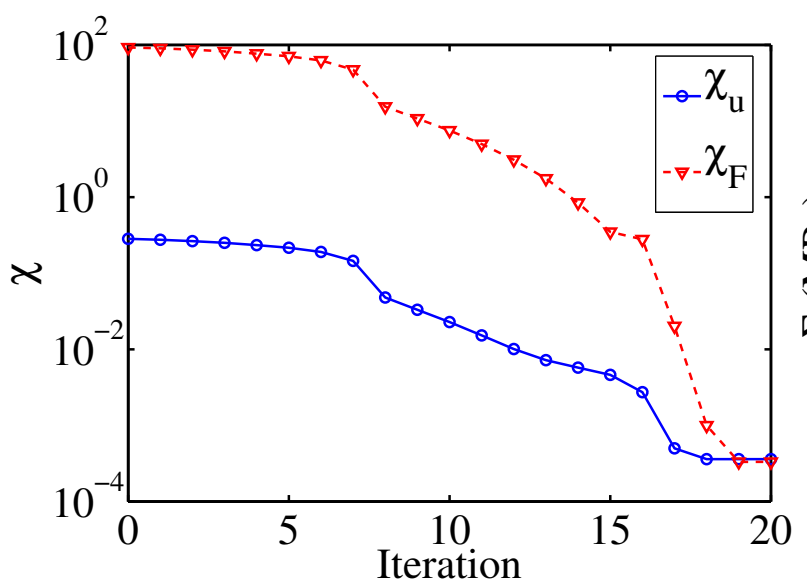

(a)

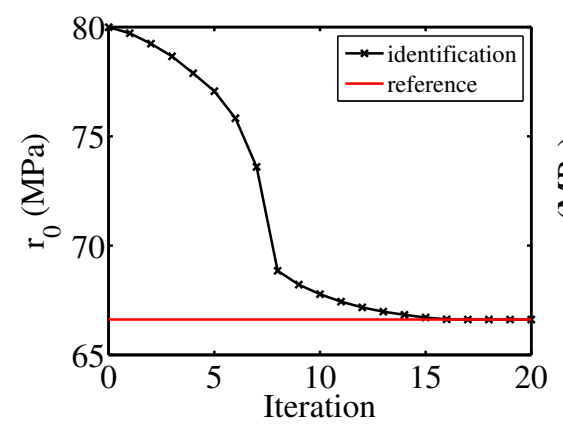

(c)

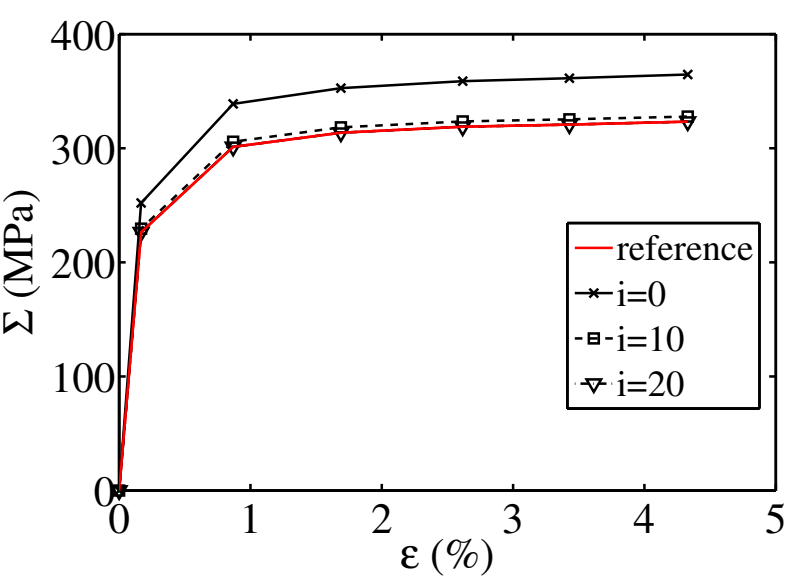

(b)

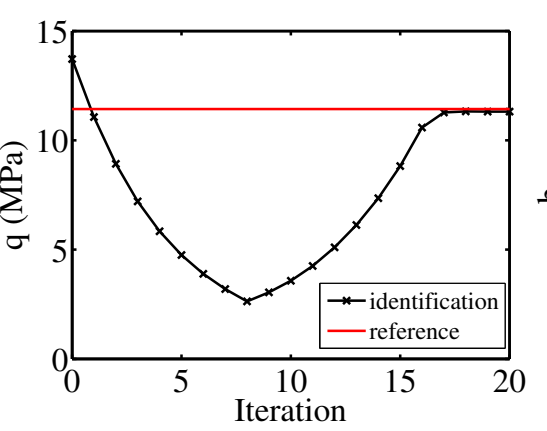

(d)

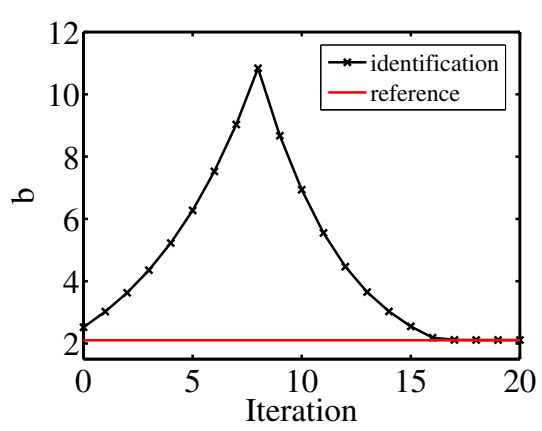

(e)

Figure 5: Cost functions $\chi_{u}$ and $\chi_{F}$ (a), macroscopic stress-strain curve (b) and parameters $r_{0}(\mathrm{c}), q$ (d) and $b$ (e), as functions of the iterations $i$ of the identification procedure performed with $w=0.5$

The results of the identification of the parameters $r_{0}$ and $b$ are shown in Figure 6 with an initial guess for the parameters that are about $50 \%$ different from the reference values. At convergence, the effective behavior is well captured and the identified parameters, $r_{0}=65.2 \mathrm{MPa}$ and $b=1.14$, are close to the reference values (i.e., $r_{0}=67 \mathrm{MPa}$ and $b=1.2$ ). The final gap between the response of Berveiller-Zaoui's model and that of the 3D calculation is low (i.e., $\chi_{F}=0.9$ ). However, the gap in terms of displacements between the two finite element calculations is rather significant and barely changes over the iterations (i.e., $\chi_{u}=14.5$ ). More precisely, during the first twenty iterations, the identification is mostly driven by $\chi_{F}$ since it is greater than $\chi_{u}$. During these first iterations $\chi_{u}$ is increasing but then decreases when $\chi_{F}$ reaches low values. At the end of the 


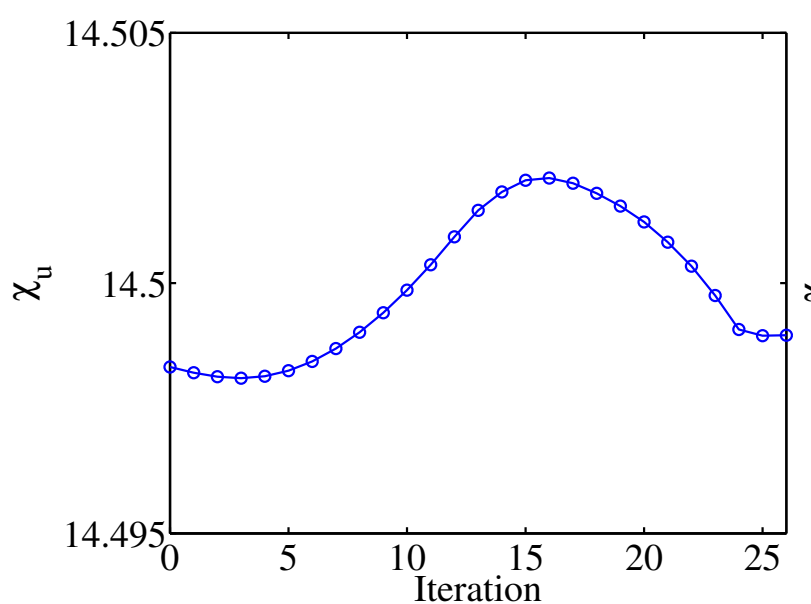

(a)

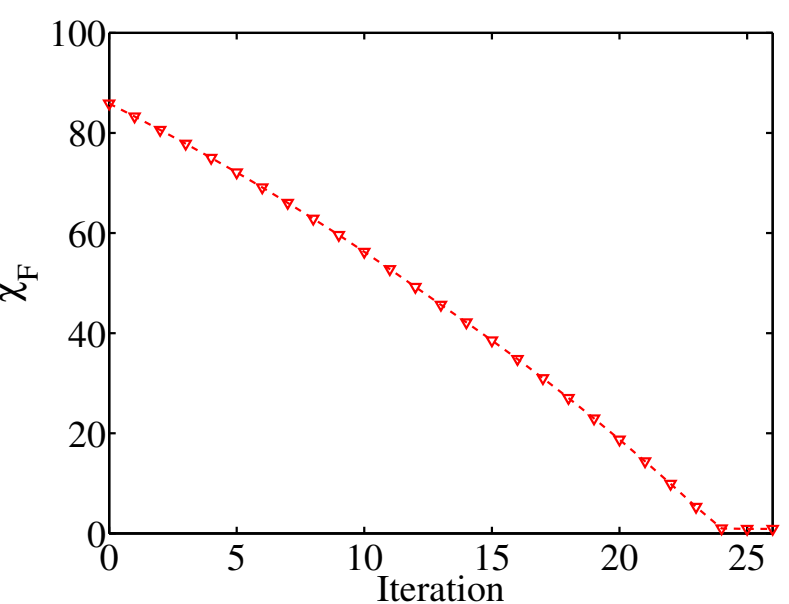

(b)

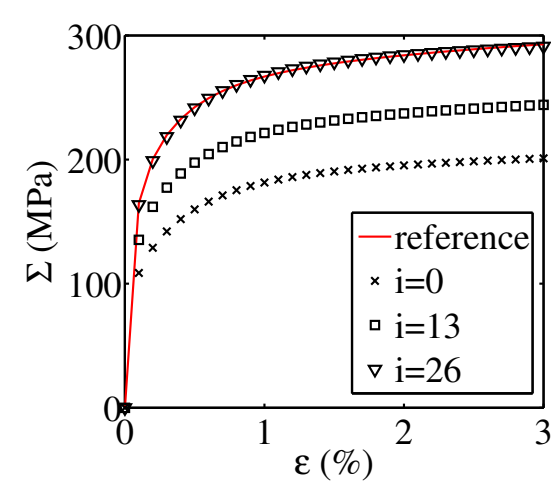

(c)

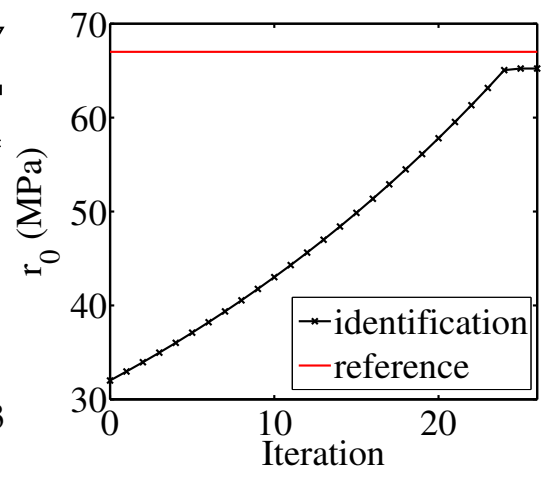

(d)

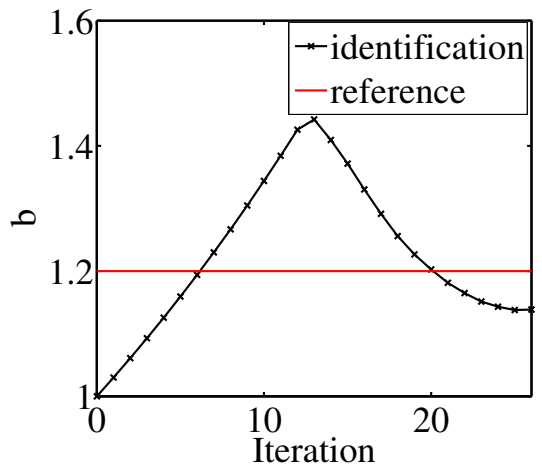

(e)

Figure 6: Cost functions $\chi_{u}$ (a) and $\chi_{F}$ (b), macroscopic stress-strain curve (c) and parameters $r_{0}$ (d) and $b$ (e), as functions of the iterations $i$ of the identification procedure performed with $w=0.5$

identification the displacement gap $\chi_{u}$ remains large as a result of the $3 \mathrm{D}$ to $2 \mathrm{D}$ approximation in the modeling when the parameters $r_{0}$ and $b$ are optimized. For instance, the displacement field along the tensile direction obtained with the $2 \mathrm{D}$ calculation at convergence is compared to the reference one in Figure 7. It is observed that local differences remain notable.

The main result of this test case is the small change of $\chi_{u}$ with the number of iterations. To better understand this result, Figure 8 shows the change of the cost functions $\chi_{u}$ and $\chi_{F}$ with the values of the parameters $r_{0}$ and $b$ about their identified values. It illustrates that $\chi_{u}$ is weakly sensitive to $r_{0}$ and $b$. Conversely, $\chi_{F}$ is very sensitive to $r_{0}$ and less to $b$. Moreover, when looking 


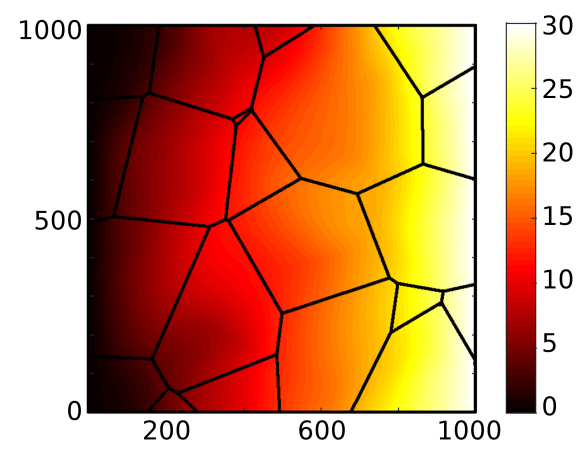

(a)

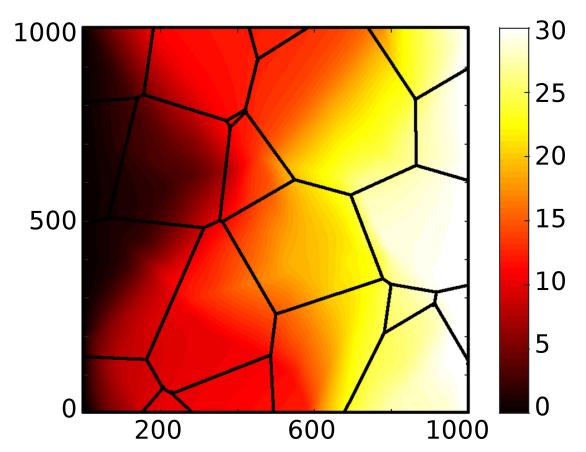

(b)

Figure 7: Displacement fields expressed in micrometers along the horizontal (tensile) direction obtained on the "observed" face, from the 3D calculation with the reference values of parameters (a) and from the 2D calculation with the identified values of parameters (b), for a macroscopic strain of $3 \%$. The spatial axes are expressed in micrometers

at the map of $\chi_{u}$, it is noted that the identified point is not a minimum of the function. Thus, by considering only this cost function, the identification would lead to small values of $b$ and high values of $r_{0}$, which would not be consistent with the reference effective behavior according to Figure $8 \mathrm{~b}$. Consequently, the combination of the two cost functions appears mandatory. Through the level of $w$, a lower weight may be given to the effective behavior to enable for a decrease of the gap in displacement fields. In the present test case, this would not allow $\chi_{u}$ to significantly change according to the dynamic range of this function (Figure 8a). However, it will be shown in the sequel that the adjustment of $w$ is key to optimize the identification.

\section{Identification results}

The numerical test cases have shown in the previous section the robustness and effectiveness of the identification procedure. An application to experimental data is now proposed.

\subsection{Reference experimental fields}

Five experimental microstructures obtained from a 316LN austenitic stainless steel, denoted $A_{70}, B_{10}, C_{10}, D_{50}$ and $E_{1000+}$, are considered with different mean grain sizes (see Table 1). 

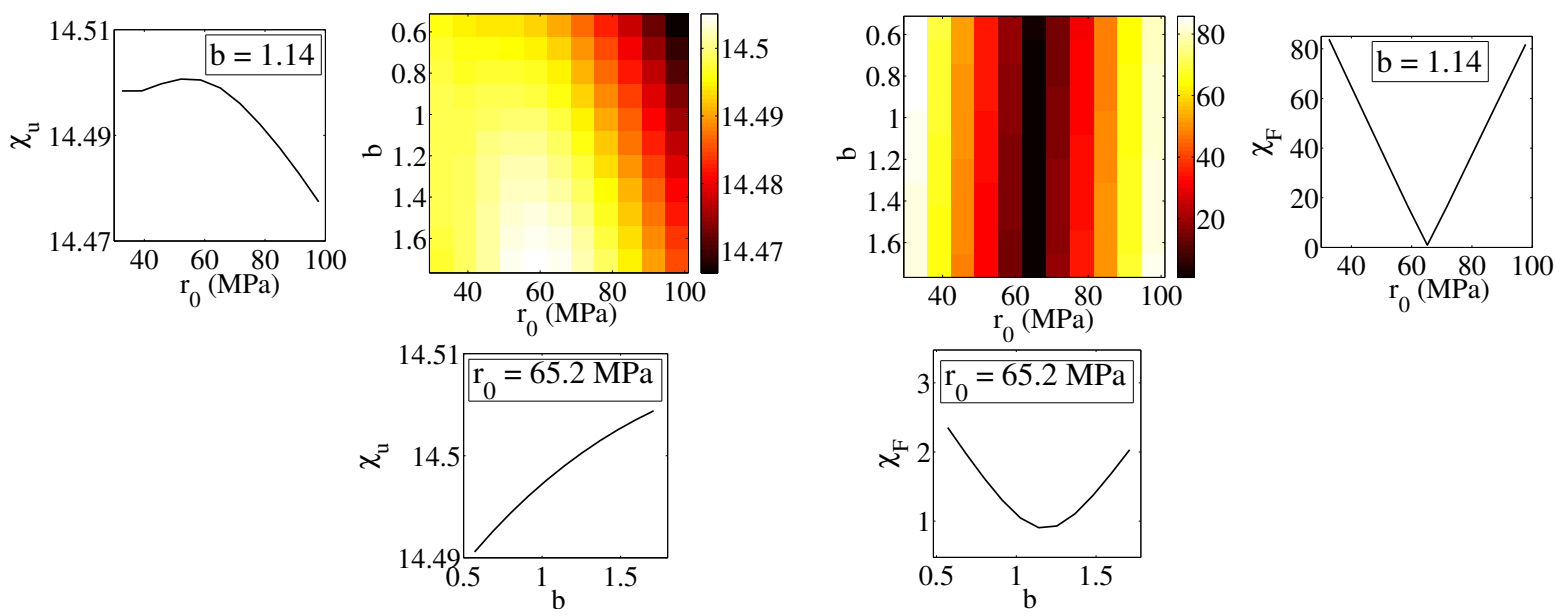

(a)

(b)

Figure 8: Cost functions $\chi_{u}$ (a) and $\chi_{F}$ (b) versus the values of the parameters $r_{0}$ and $b$ when the reference values are $r_{0}=67 \mathrm{MPa}$ and $b=1.2$

The microstructures are shown in Figure 9. Kinematic measurements are performed on their surface by DIC in ROIs of different sizes and from a sequence of SEM images acquired during in situ tensile tests [3]. The corresponding macroscopic stress-strain curves are presented in Figure 10. The displacement uncertainties for each specimen are given in Table 1. Figure 11 shows the displacement fields along the horizontal (tensile) direction for the five microstructures measured for a mean tensile strain of the order of $4 \%$. The two components of the displacement fields with their time evolution up to $4 \%$ are used in the following identification procedure as

Table 1: DIC standard displacement uncertainties for the five studied microstructures

\begin{tabular}{|c|c|c|c|}
\hline Specimen & Mean grain size $(\mu \mathrm{m})$ & ROI size $\left(\mu \mathrm{m}^{2}\right)$ & Displacement uncertainty $(\mathrm{nm})$ \\
\hline$A_{70}$ & 70 & $200 \times 200$ & 4.3 \\
\hline$B_{10}$ & 10 & $200 \times 200$ & 5.9 \\
\hline$C_{10}$ & 10 & $100 \times 100$ & 2.2 \\
\hline$D_{50}$ & 50 & $400 \times 400$ & 7.1 \\
\hline$E_{1000+}$ & $>1000$ & $400 \times 400$ & 4.4 \\
\hline
\end{tabular}




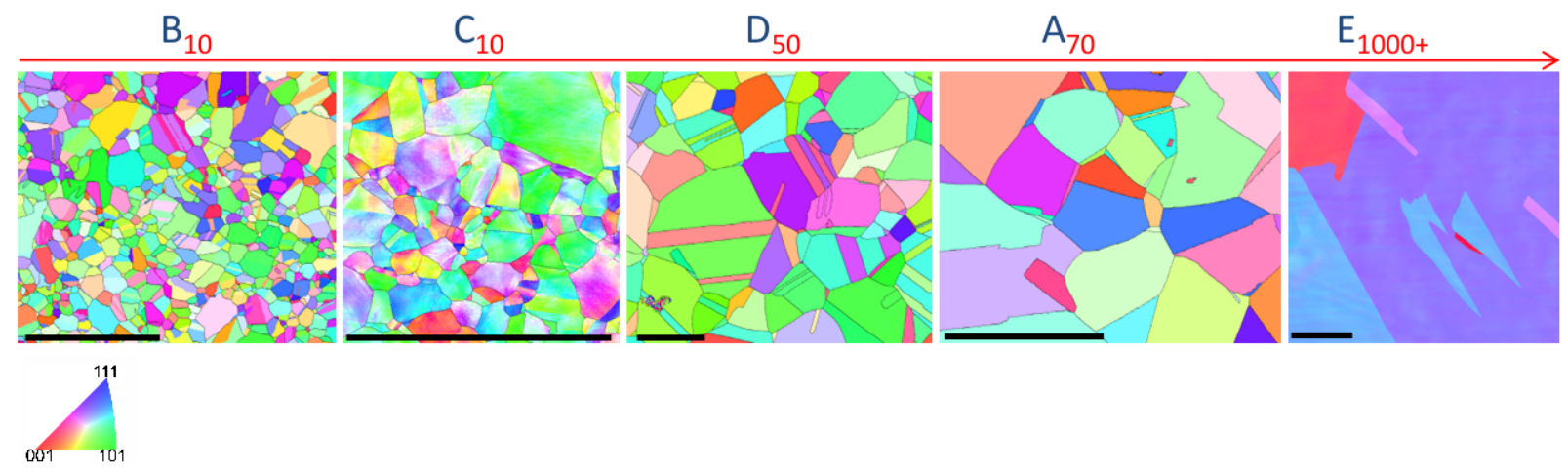

Figure 9: IPF of the the five studied microstructures sorted in increasing mean grain size order. The scale bar is $100 \mu \mathrm{m}$ long

the (reference) experimental fields.

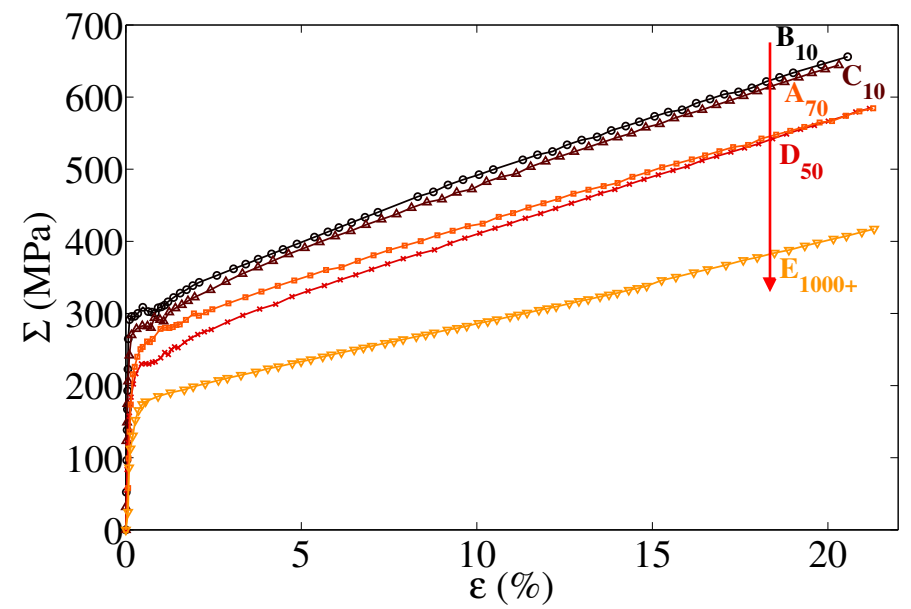

Figure 10: Experimental macroscopic stress-strain curve of the in situ tensile tests for the the five studied microstructures

From monotonic tensile tests, only parameters associated with the isotropic hardening part of the crystal plasticity law (i.e., $r_{0}, b$ and $q$; see AppendixA) will be identified. One may also consider the coefficients $h_{s r}$ of the interaction matrix between slip systems used in the isotropic hardening equation. These parameters will also be investigated in the following. To identify the parameters associated with kinematic hardening (i.e., $c$ and $d$ ), cyclic tests are required. 


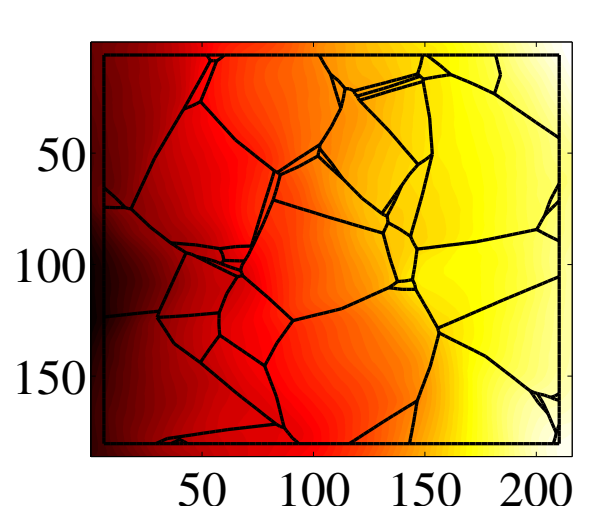

(a)

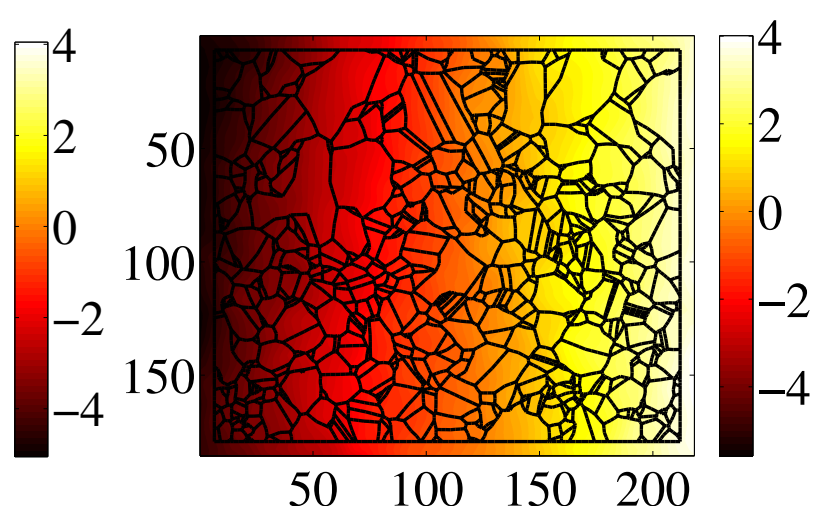

(b)

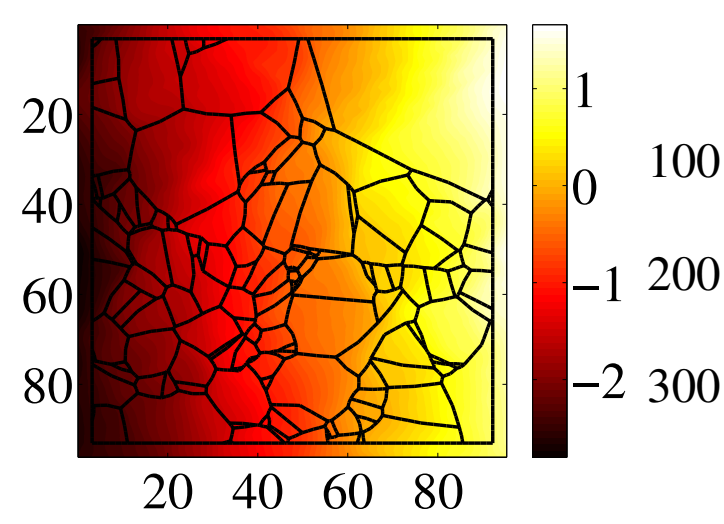

(c)

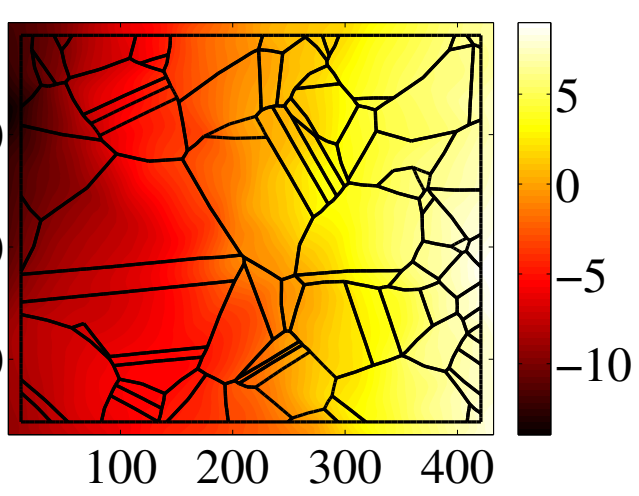

(d)

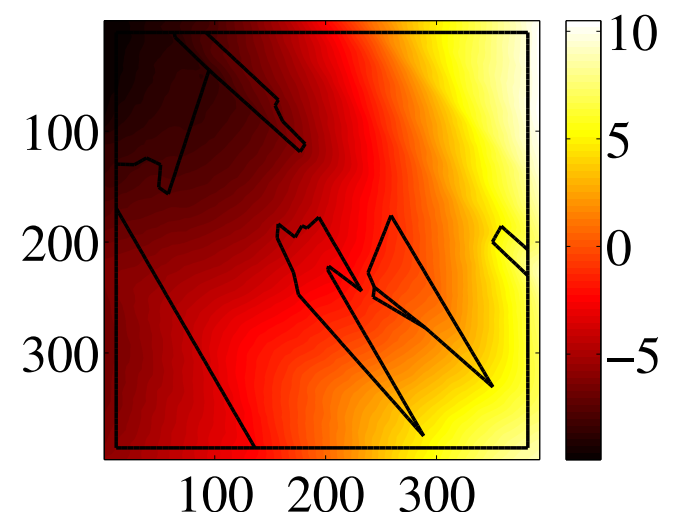

(e)

Figure 11: Displacement fields expressed in micrometers measured by DIC along the horizontal (tensile) direction for a mean tensile strain of $4 \%$, for the microstructures denoted $A_{70}$ (a), $B_{10}(\mathrm{~b}), C_{10}$ (c), $D_{50}$ (d) and $E_{1000+}$ (e). The microstructure boundaries are shown as black lines on these fields 


\subsection{Isotropic hardening parameters}

It is first proposed to identify the sought isotropic hardening parameters $r_{0}, b$ and $q$. The sensitivity of the computed displacement fields $\boldsymbol{u}_{c}$ and average stress $\boldsymbol{F}_{c}$ to each considered parameter is first assessed, focusing on the case of specimen $A_{70}$. A nonzero sensitivity is a necessary condition for making the identification possible. It is assessed by calculating the difference of the simulated response involved by a chosen variation of each parameter. Figure 12 shows the sensitivity of $\boldsymbol{u}_{c}$ to $r_{0}, b$ and $q$ for a mean tensile strain of the order of $4 \%$. It corresponds to a (forward) variation of $20 \%$ of each parameter from the values identified by homogenization (given in Table A.5), the other parameters being fixed (Table A.6). It is noted that the sensitivity fields are identical for a variation of either $b$ or $q$, but different from a variation of $r_{0}$.

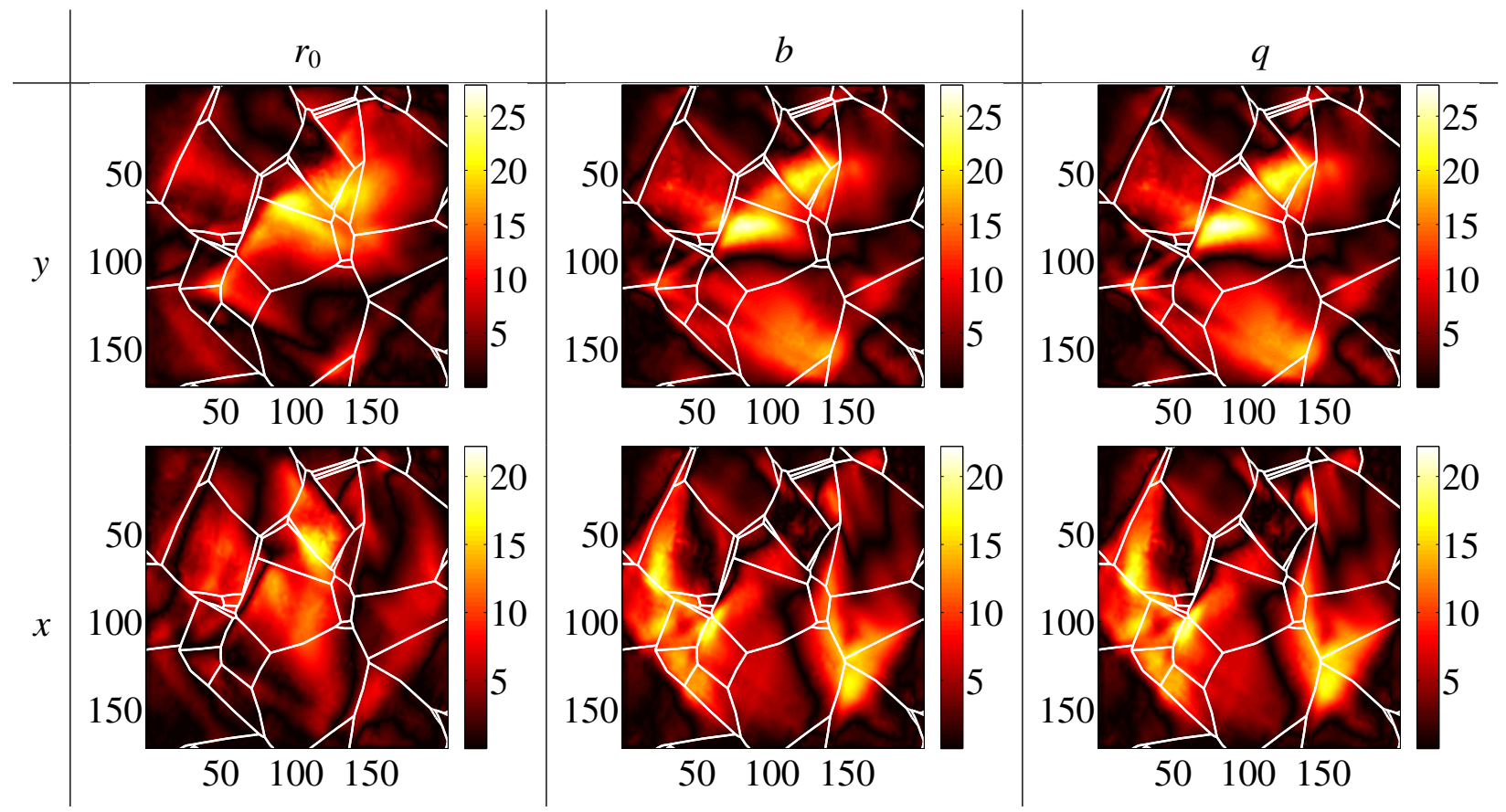

Figure 12: Absolute value of the sensitivity of the simulated displacement field $\left|\boldsymbol{\delta} \boldsymbol{u}_{c}\right|$ (expressed in nanometers), for a $20 \%$ (forward) variation of each of the parameters $r_{0}, b$ and $q$ shown along the horizontal $y$ and vertical $x$ directions for a mean strain of about $4 \%$. The spatial axes are expressed in micrometers

This result is valid throughout the loading history as shown in Figure 13a. Thus, $b$ and $q$ cannot be identified separately from the displacement fields, nor from the macroscopic behavior 
according to Figure $13 \mathrm{~b}$ showing the sensitivity of the load calculated by homogenization with respect to each parameter. Only $r_{0}$ and the product $q b$ are chosen to be identified in the sequel.

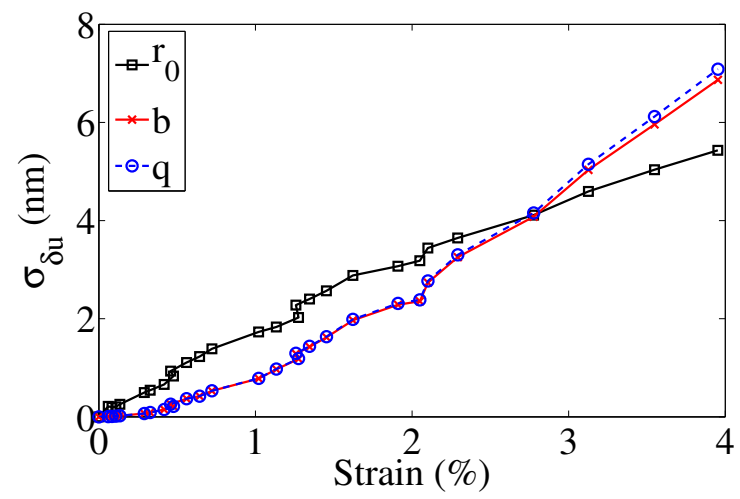

(a)

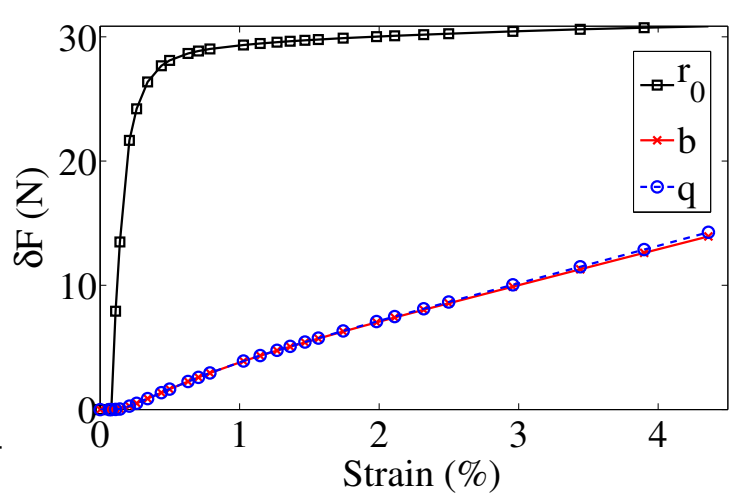

(b)

Figure 13: Root mean square value of the field $\boldsymbol{\delta} \boldsymbol{u}_{c}$ (a) and sensitivity of the load calculated by homogenization (b) corresponding to a $20 \%$ variation of each of the parameters $r_{0}, b$ and $q$ as functions of the mean strain

Moreover, it is observed that the fields $\delta \boldsymbol{u}_{c}$ change significantly from grain to grain and the highest values are generally reached close to grain boundaries. A given microstructure can potentially be more or less favorable to the identification of the parameters. However, among the five studied microstructures, no significant differences on the sensitivity fields have been noticed. It is worth noting that the sensitivity of the displacement fields to $r_{0}$ is higher than that to $b$ or $q$ in the first part of the loading (i.e., for a mean tensile strain less than $2.8 \%$ ), then the opposite trend occurs (Figure 13a). It is related to the role of these parameters with the yield condition and with the hardening rate, respectively. At the same time, the macroscopic load is much more sensitive to $r_{0}$ than to $b$ or $q$ (Figure 13b).

It is interesting to note that $\boldsymbol{\delta} \boldsymbol{u}_{c}$ is locally several times higher than the measurement resolution (4.3 $\mathrm{nm}$ for the investigated microstructure, see Table 1). However, the root mean square of $\boldsymbol{\delta} \boldsymbol{u}_{c}$ is greater than the measurement uncertainty only for the last three time steps (Figure 13a). The displacement fields associated with high levels of average strain will thus contribute to the parameter identification. Furthermore, as reported in other studies [7, 8], it is possible to a priori estimate the uncertainty of the identified parameters related to the DIC measurement uncertainty 
corresponding to SEM noise. Only the displacement fields are considered in this analysis (i.e., $w=0$ ). According to Equation (8), a variation of the displacement fields $\{\boldsymbol{\delta} \boldsymbol{u}\}$ from the converged value implies a variation of parameters $\{\boldsymbol{\delta} \boldsymbol{p}\}$ such that

$$
\{\boldsymbol{\delta} \boldsymbol{p}\}=\left[\boldsymbol{H}_{u}\right]^{-1}\left(\sum_{t}\left[\boldsymbol{S}_{u}\right]_{t}^{T}[\boldsymbol{M}]\{\boldsymbol{\delta} \boldsymbol{u}\}_{t}\right)
$$

Assuming that $\{\boldsymbol{\delta} \boldsymbol{u}\}$ is only due to the imaging noise and time-independent, from the expression of the covariance matrix $\left[\mathbf{C o v}_{u}\right]$ of the measured kinematic degrees of freedom (Equation (5)), the covariance matrix $\left[\boldsymbol{C o v} \boldsymbol{v}_{p}\right]=\langle\{\delta \boldsymbol{p}\} \otimes\{\boldsymbol{\delta} \boldsymbol{p}\}\rangle$ of the identified parameters reads

$$
\left[\boldsymbol{C o v}_{\boldsymbol{p}}\right]=2 \eta_{f}^{2}\left[\boldsymbol{H}_{u}\right]^{-1}\left(\sum_{t}[\boldsymbol{P}]_{t}[\boldsymbol{M}]^{-1}[\boldsymbol{P}]_{t}^{T}\right)\left[\boldsymbol{H}_{u}\right]^{-1}
$$

with

$$
[\boldsymbol{P}]_{t}=\left[\boldsymbol{S}_{u}\right]_{t}^{T}[\boldsymbol{M}]
$$

It is evaluated in the case of the specimen $A_{70}$ as

$$
\left[\boldsymbol{C o v}_{p}\right]=\left[\begin{array}{ccc}
0.0018 & -0.0001 & 0.032 \\
-0.0001 & 0.0002 & -0.040 \\
0.032 & -0.040 & 8.4
\end{array}\right]
$$

when $\{\boldsymbol{p}\}=\left\{\begin{array}{lll}r_{0} & b & q\end{array}\right\}^{T}$ ( $r_{0}$ and $q$ are to be expressed in MPa while $b$ is dimensionless). The diagonal terms correspond to the variance of each parameter considered separately. It is observed that the variance on these three parameters is low. It means that the identification procedure proposed in this study shows little sensitivity to SEM imaging noise. The off-diagonal coefficients are clearly not negligible indicating a coupling between parameters. To better highlight this result, one may use the correlation matrix $[\boldsymbol{R}]$ defined as

$$
R_{i j}=\frac{\operatorname{Cov}_{p_{i j}}}{\sqrt{\operatorname{Cov}_{p_{i i}} \operatorname{Cov}_{p_{j j}}}}
$$

The value of $R_{i j}$ is equal to 1 when the parameters $i$ and $j$ are correlated, -1 when anti-correlated, and 0 when uncorrelated. The corresponding matrix reads 


$$
[\boldsymbol{R}]=\left[\begin{array}{ccc}
1 & -0.24 & 0.26 \\
-0.24 & 1 & -0.998 \\
0.26 & -0.998 & 1
\end{array}\right]
$$

This result shows that $b$ and $q$ are strongly coupled, which is consistent with the previous observations (Figures 12 and 13a). More precisely, they are anti-correlated. Since $d(q b) /(q b)=$ $d q / q+d b / b$, if $d q / q$ and $d b / b$ have opposite trends (i.e., $d q / q=-d b / b$ ) their normalized cross correlation is -1 , and $d(q b)$ vanishes. Moreover, the coupling between $r_{0}$ and $b$ or $q$ is low but non-zero thereby indicating a rather low correlation.

The identification of the parameters $r_{0}$ and $q b$ (i.e., $b$ with $q$ fixed) is first performed for specimen $A_{70}$ and for several values of the weight $w$. The values identified by homogenization (i.e., for $w=1$, given in Table A.5) are used as an initial guess of the parameters. The other parameters of the law are kept fixed to the reference values (Table A.6). When $w=1 / 2$, the identification procedure leads to negligible changes of the cost functions and of the parameter values. The minimization procedure seems to be locked in a minimum point and the cost function dealing with the displacement fields appears to bring no added value. Lower weights given to the macroscopic behavior are then considered (i.e., 0.1, 0.01, 0.001). In these cases, Figure 14 shows the change of the cost functions $\chi_{u}$ and $\chi_{F}$ with the iteration number of the identification procedure. As expected, the lower the weight, the greater the gap on the effective behavior and the lower the gap on the displacement fields. Starting from 21.8, a minimum of 18.3 is reached for $\chi_{u}$ with $w=0.001$. A minor difference is however observed between $w=0.01$ and $w=0.001$. The variation of $w$ has a much stronger effect on $\chi_{F}$.

The corresponding change of the simulated effective behavior is presented in Figure 15. Decreasing $w$ allows the homogenized behavior to deviate from the experimental curve. If the simulated stress-strain curves obtained with $w=0.01$ or $w=0.001$ do not appear realistic, the gap between the simulated curve obtained with $w=0.1$ and the experimental data remains acceptable. This is subjective. An acceptance criterion may be investigated in future studies considering for example a confidence indicator in the homogenization model [21].

The identified values of the parameters $r_{0}$ and $b$ are shown in Figure 16 as functions of 


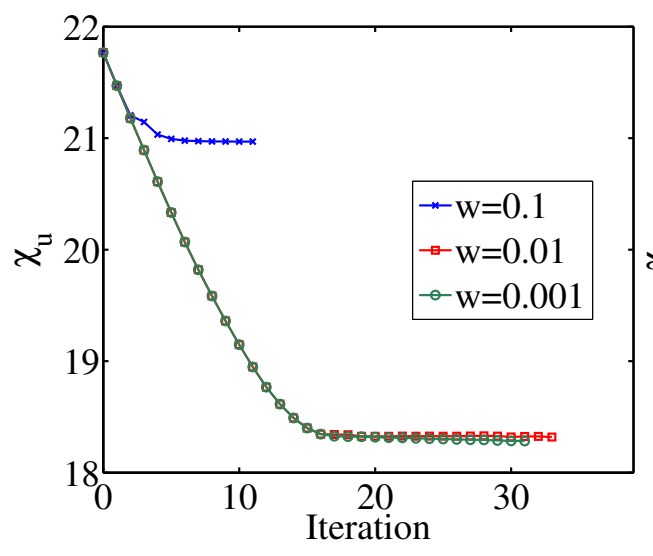

(a)

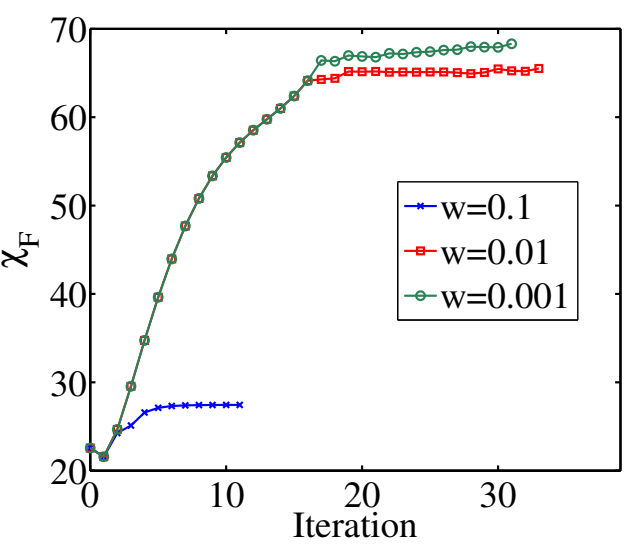

(b)

Figure 14: Changes of the cost functions $\chi_{u}$ (a) and $\chi_{F}$ (b) with the iteration number of the identification procedure performed with $w=0.1, w=0.01$ and $w=0.001$

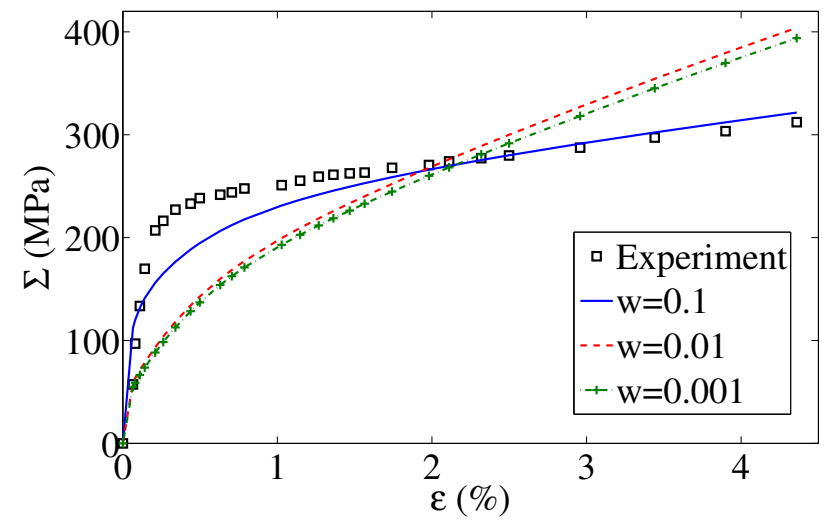

Figure 15: Comparison between the stress-strain curves obtained experimentally and those obtained by homogenization at convergence of the identification procedure performed with $w=0.1, w=0.01$ and $w=0.001$

the value of the weight $w$. A range of values is obtained, bounded by the extrema obtained by considering only the macroscopic stress (i.e., for $w=1$ ) and microscopic (i.e., for $w=0$ ) displacement fields.

Regarding the displacements, the influence of $w$ on the gap between the measured and simulated fields is shown in Figure 17. In order to underline the influence of the whole loading history, the sum of the gaps over all the time steps is presented in this figure, which is consistent 


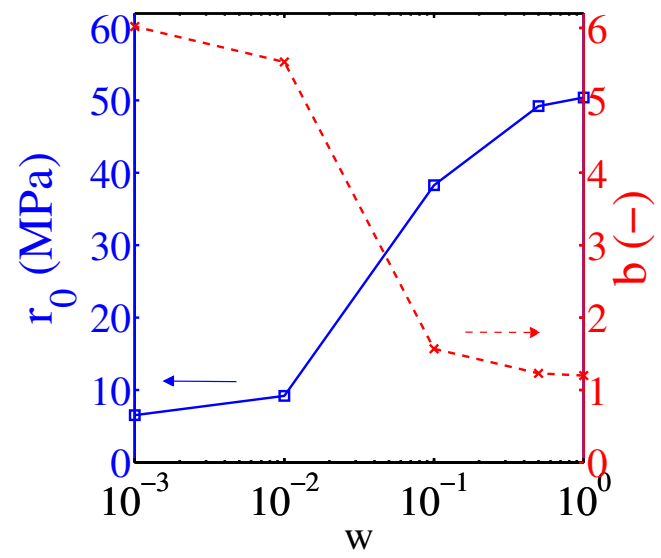

Figure 16: Values of the identified parameters $r_{0}$ and $b$ as functions of the value of $w$

with the cost function $\chi_{u}$. The same displacement dynamic range is used to make the comparison easier. The identification leads to a decrease of the gap over the displacement fields for either value of $w$. This gap is reduced even further if a low value of $w$ is chosen. One may note that the gap is still significant when convergence is complete, even if only $\chi_{u}$ is considered (i.e., $w=0$ ). In that case, the gap is virtually identical to that obtained when $w=0.001$. This residual gap, much higher than the measurement uncertainty, corresponds to the error of the polycrystalline model once the parameters associated with isotropic hardening of the crystal plasticity law have been optimized. 


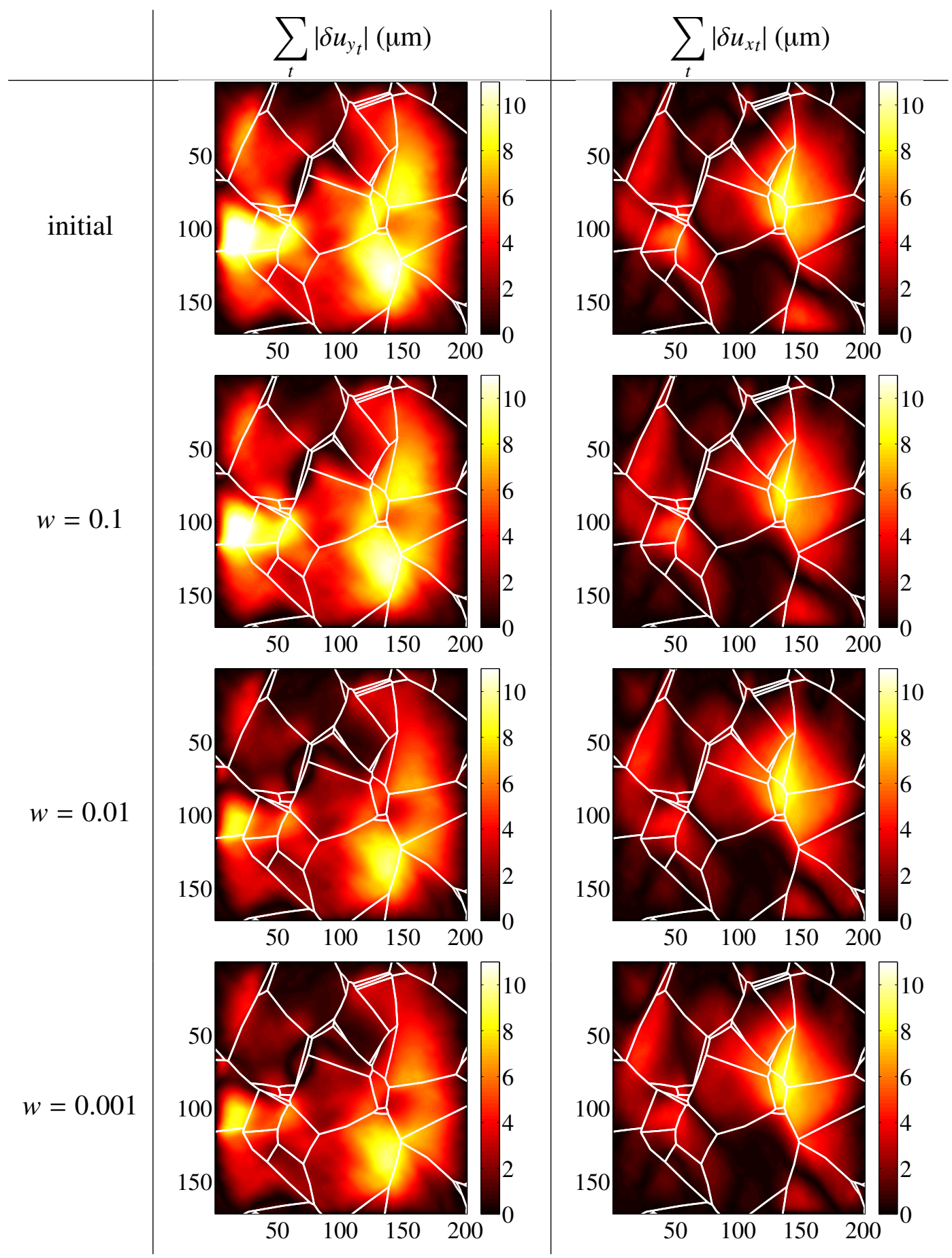

Figure 17: Sum over all time steps of the absolute value of the difference between displacements fields along the horizontal and vertical, when measured by DIC and simulated before and after the identification procedure is performed with $w=0.1, w=0.01$ and $w=0.001$. The spatial axes are expressed in micrometers 
The influence of the mean grain size on the identified parameters is assessed. For that purpose the four experimental microstructures $C_{10}, D_{50}, A_{70}$ and $E_{1000_{+}}$, whose measured mean grain size is respectively $10 \mu \mathrm{m}, 50 \mu \mathrm{m}, 70 \mu \mathrm{m}$ and millimetric, are considered [3]. For each microstructure, the identification of $r_{0}$ and $q b$ is performed for $w=0.1$ since this weight is identified in the case of $A_{70}$ as the best trade-off to reduce $\chi_{u}$ while obtaining a satisfactory simulated effective behavior (Figures 14 and 15). The identified values are reported in Table 2. A slight change of the parameter levels is observed when compared to their reference values (identified with $w=1$ ). Moreover, the decrease of the critical resolved shear stress $r_{0}$ with an increase of the mean grain size is observed both on the initial values and at convergence of the optimization procedure. This trend is associated with the Hall-Petch effect and is consistent with the experimental observations [3].

Table 2: Identified values of the parameters associated with isotropic hardening when $w=0.1$ for different mean grain sizes

\begin{tabular}{|c|c|c|c|c|c|c|c|}
\cline { 2 - 8 } \multicolumn{1}{c|}{} & \multicolumn{2}{c|}{$r_{0}(\mathrm{MPa})$} & \multicolumn{2}{c|}{$b(-)$} & $q(\mathrm{MPa})$ & \multicolumn{2}{c|}{$q b(\mathrm{MPa})$} \\
\hline Specimen & reference & identified & reference & identified & reference & reference & identified \\
\hline$C_{10}$ & 60 & 56 & 1.20 & 1.27 & 195 & 234 & 248 \\
\hline$D_{50}$ & 41 & 42 & 1.20 & 1.17 & 179 & 215 & 209 \\
\hline$A_{70}$ & 50 & 38 & 1.20 & 1.57 & 163 & 196 & 254 \\
\hline$E_{1000+}$ & 29 & 30 & 0.40 & 0.38 & 159 & 64 & 61 \\
\hline
\end{tabular}

If the Hall-Petch relationship is usually identified at the macroscopic scale from the yield stress dependence on the mean grain size, it is proposed herein to identify it at the slip system scale from the critical resolved shear stress. For that purpose, a relationship between $r_{0}$ and the mean grain size $d$ is sought such that

$$
r_{0}=c_{1}+\frac{c_{2}}{\sqrt{d}}
$$

where $c_{1}$ is a constant and $c_{2}$ is the Hall-Petch factor. The results of the least squares fit are presented in Figure 18 for the identified values of $r_{0}$ when $w=1$ and $w=0.1$. It is observed 
that the choice $w=0.1$ for the identification leads to the best fit of Hall-Petch's law with a correlation coefficient of 0.99 against 0.91 when $w=1$. In that case $c_{1}=28 \mathrm{MPa}$ and $c_{2}=$ $0.09 \mathrm{MPa} \cdot \mathrm{m}^{1 / 2}$. To the best of the authors' knowledge, no similar identification results of HallPetch's law at the slip systems scale are reported in the literature. The fact that a better agreement is found than by using macroscopic data tends to validate the choice of $w=0.1$ to optimize the simulated displacement fields. This application of the identification method from experimental fields then leads to a new set of parameters associated with isotropic hardening with an improved representativity of the polycrystalline behavior.

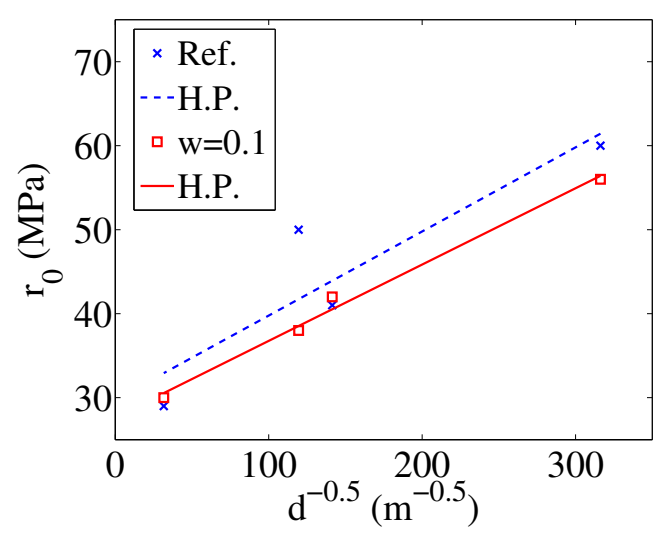

Figure 18: Identification of the Hall-Petch (H.P.) law from the values of the initial critical resolved shear stress $r_{0}$ (dots) identified by homogenization and from the ones identified when $w=0.1$

\subsection{Coefficients of the interaction matrix between slip systems}

It is now proposed to identify the coefficients of the interaction matrix between slip systems. These coefficients currently remain poorly known and difficult to identify via experimental or numerical investigations. For example, conducting tedious and numerous dislocation dynamics simulations is one way of obtaining such coefficients [22]. Since the interactions between slip systems should be associated with variations of the polycrystalline material response at the microstructural scale, it is interesting to apply the present identification method to these coefficients.

As only local microstructural kinematic effects are considered and not the effective response, the weight $w=0$ is chosen to perform the identification. Such an identification, which is only 
based on measured displacement fields, is possible since they are sensitive to a (forward) variation of each coefficient as shown in Figure 19 in the case of specimen $A_{70}$. It is observed that the displacement fields at the last time step (i.e., for a mean tensile strain of about $4 \%$ ) are particularly sensitive to a variation of $h_{5}, h_{4}$ and $h_{1}$. This is true throughout the loading history, which is quantified by considering the root mean square difference of displacement fields at every time step (Figure 20a). As for $r_{0}, q$ and $b$, only a part of the displacement fields contributes to the identification of the coefficients, where $\delta \boldsymbol{u}_{c}$ is higher than the standard displacement resolution (4.3 $\mathrm{nm}$ for this microstructure, see Table 1).

Moreover, the correlation between the coefficients is assessed prior to running the identification procedure. The correlation matrix $[\boldsymbol{R}]$ is calculated considering a white Gaussian noise and $\{\boldsymbol{p}\}=\left\{\begin{array}{llllll}h_{1} & h_{2} & h_{3} & h_{4} & h_{5} & h_{6}\end{array}\right\}^{T}$. The corresponding matrix is plotted in Figure 21a. The absolute value of $[\boldsymbol{R}]$ is also given in Figure $21 \mathrm{~b}$ to highlight the various correlations. The selfhardening coefficient $h_{1}$ and the coplanar interaction coefficient $h_{2}$ are uncorrelated. In addition, the coefficient $h_{5}$ appears almost uncorrelated with $h_{1}, h_{2}$ and $h_{3}$. Conversely, $h_{2}$ and $h_{3}$ are strongly correlated. As a consequence, $h_{1}, h_{2}, h_{4}$ and $h_{5}$ are chosen to be identified because they involve the highest sensitivity of the displacement fields and they are sufficiently uncorrelated.

However, arbitrary variations of these coefficients translate into changing the macroscopic loading curve (Figure 20b). Thus, to ensure that the identification based only on the displacement fields lead to a realistic simulated effective behavior, a particular combination of the coefficients that keeps the simulated homogeneous load level unchanged is desirable. It is helpful to consider the Hessian matrix $\left[\boldsymbol{H}_{F}\right]$ defined in Equation (10) and assessed from the reference values of the parameters (i.e., identified by homogenization). The eigen vectors corresponding to the two highest eigen values of $\left[\boldsymbol{H}_{F}\right]$ express the two directions for searching the parameters leading to the highest variation of the simulated homogeneous load level. The coefficients are then chosen to be sought in the subspace orthogonal to these two directions. This provides the first two equations of system (20). Since the coefficient $h_{1}$ is directly involved in the isotropic hardening rate, it is chosen to keep it fixed to its reference value. Thus, the full system of equations preventing the variations of the effective behavior reads 

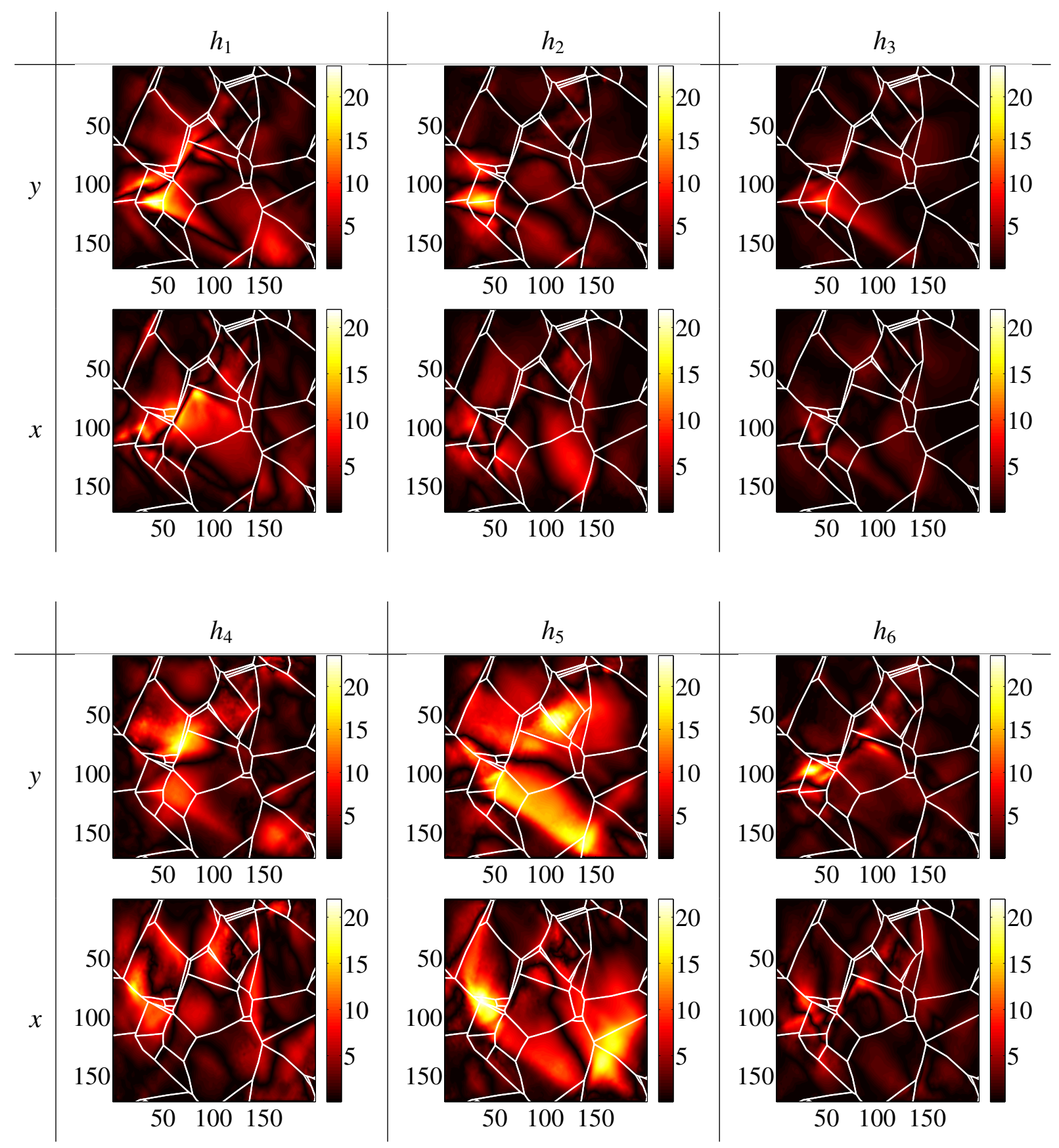

Figure 19: Absolute value of the sensitivity of the simulated displacement field $\left|\boldsymbol{\delta} \boldsymbol{u}_{c}\right|$ (expressed in nanometers), for a $20 \%$ (forward) variation of each of the coefficients of the interaction matrix shown along the horizontal $y$ and vertical $x$ directions for a mean strain of about $4 \%$. The spatial axes are expressed in micrometers 


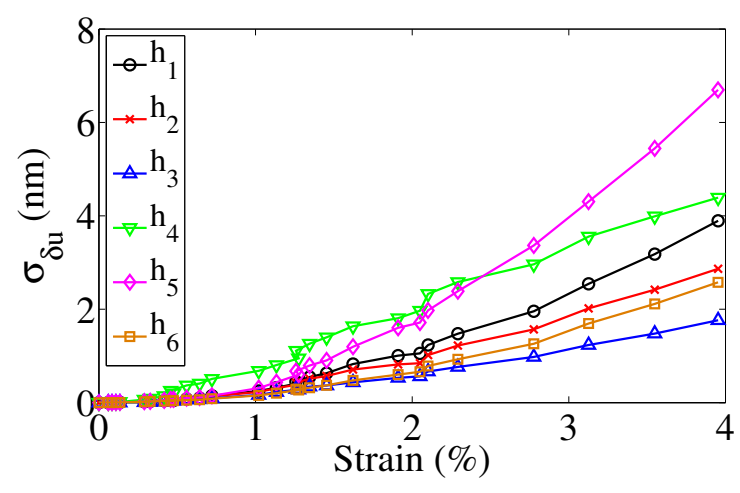

(a)

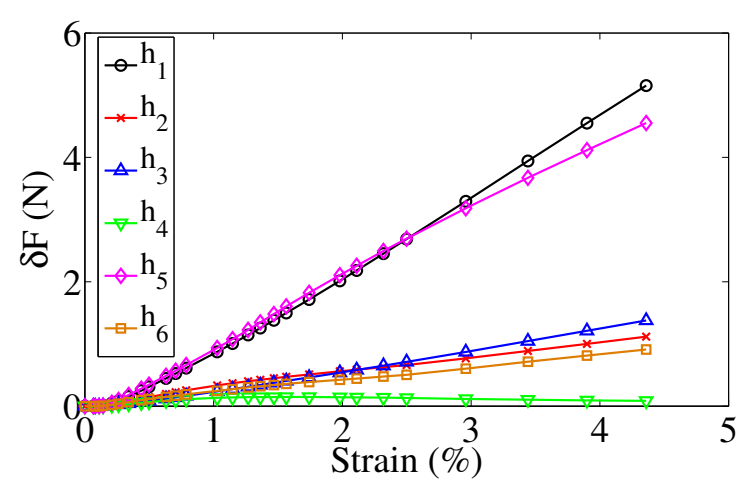

(b)

Figure 20: Root mean square value of the field $\boldsymbol{\delta} \boldsymbol{u}_{c}$ (a) and sensitivity of the load calculated by homogenization (b) corresponding to a $20 \%$ (forward) variation of each of the coefficients of the interaction matrix as functions of the mean strain

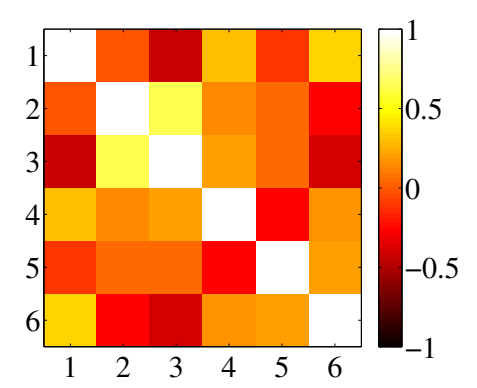

(a)

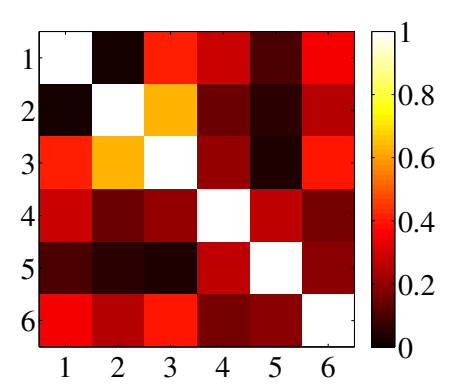

(b)

Figure 21: Correlation matrix $[\boldsymbol{R}]$ of the coefficients $h_{i}$ (a) and its absolute value (b). The axes correspond to the index $i$

$$
\left\{\begin{array}{r}
0.02 \delta h_{2}+0.37 \delta h_{3}-0.89 \delta h_{4}-0.01 \delta h_{5}+0.27 \delta h_{6}=0 \\
0.22 \delta h_{2}+0.81 \delta h_{3}+0.38 \delta h_{4}-0.38 \delta h_{5}+0.06 \delta h_{6}=0 \\
\delta h_{1}=0
\end{array}\right.
$$

The identification of the interaction matrix is then performed by minimizing $\chi_{u}$ with respect to $h_{2}, h_{4}$ and $h_{5}$, the other coefficients being set by system (20) and the other constitutive parameters being fixed to their reference values. Figure 22 shows the results of the identification. The cost function has been reduced by 0.22 , which is relatively small. One may note that the identified 
values of the coefficients are virtually constant during 10 iterations. The components of the whole interaction matrix are given and compared to their initial values in Table 3.

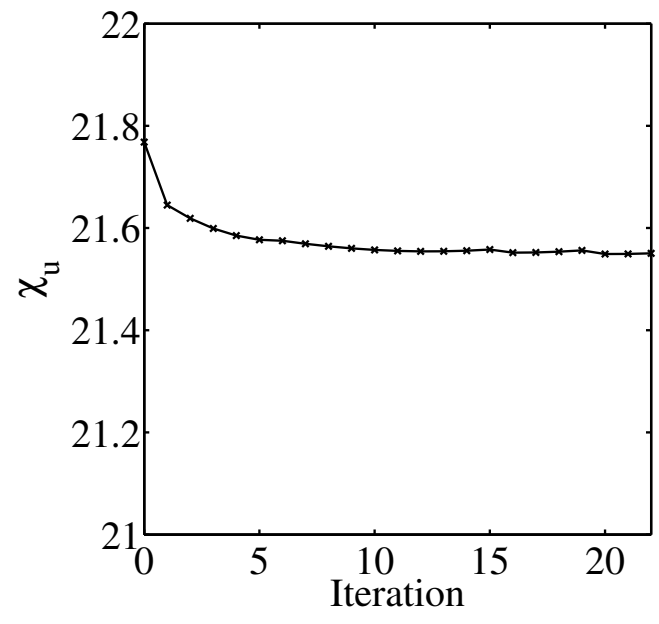

(a)

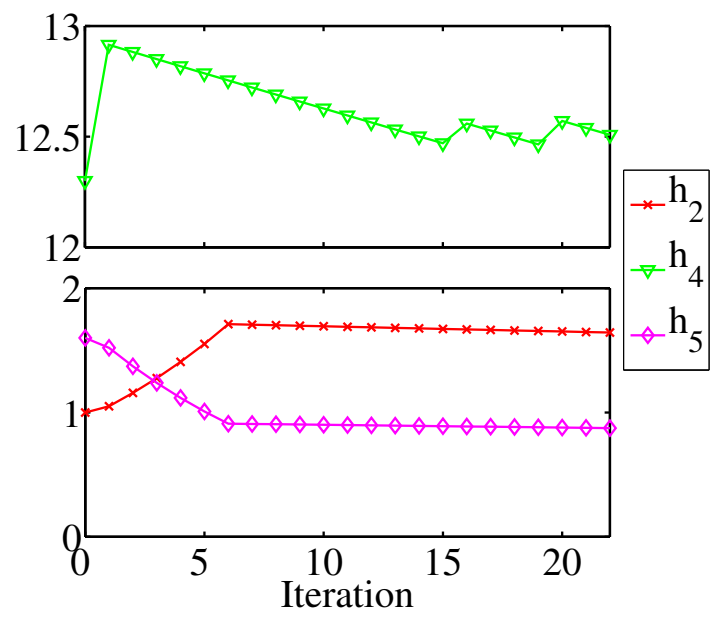

(b)

Figure 22: Cost function $\chi_{u}$ (a) and identified coefficients of the interaction matrix (b) as functions of the iterations of the identification procedure

Several observations can be made. First, the coefficient $h_{4}$, which is related to collinear interactions, barely increases and remains predominant. Then, the weight of coplanar interactions $\left(h_{2}\right)$ increases and now differs from self-hardening interactions $\left(h_{1}\right)$. Conversely, the initial slight predominance of $h_{5}$ accounting for the glissile junction is not observed anymore at convergence. Last, a large increase of $h_{6}$, which is related to Lomer's lock, is observed and a strong decrease of $h_{3}$, which is related to Hirth's lock. Given the small number of studies on the coefficients of the interaction matrix, the present approach appears as original.

Table 3: Identified values of the coefficients of the interaction matrix in the case of the specimen $A_{70}$

\begin{tabular}{|c|c|c|c|c|c|c|}
\cline { 2 - 7 } \multicolumn{1}{c|}{} & $h_{1}(-)$ & $h_{2}(-)$ & $h_{3}(-)$ & $h_{4}(-)$ & $h_{5}(-)$ & $h_{6}(-)$ \\
\hline Initial value & 1.00 & 1.00 & 0.60 & 12.3 & 1.60 & 1.30 \\
\hline Identified value & 1.00 & 1.64 & 0.10 & 12.5 & 0.87 & 2.60 \\
\hline
\end{tabular}

The inverse method proposed herein has been applied to the identification of the coefficient 
of the interaction matrix minimizing the gap on the displacement fields and keeping the predicted effective behavior realistic. This first identification provides valuable information on the relative weights of each coefficients leading to the optimized simulation of the kinematic fields at the microstructural scale.

\section{Conclusion and perspectives}

An inverse method is proposed based on both microstructural displacement fields and macroscopic stress in a combined cost function. The reason for this choice is due to the fact that microscopic displacement fields do not span over the whole width of the sample. Further, the microstructural information obtained via EBSD is only available on the surface of the sample. The present case is therefore complex and prone to model errors (e.g., partially known boundary conditions, constitutive law error). This leads to a weighting between the two data sets (i.e., displacement field and applied load) to be altered with respect to a consistent probabilistic treatment.

Once the weight associated with the total cost function is chosen, it is shown by a sensitivity analysis that only two isotropic hardening coefficients can be identified for studied law, and three parameters of the interaction matrix associated with slip systems. This a priori analysis is possible thanks to the knowledge of the measurement uncertainties [3] and the present setting with a finite element model updating framework. Four different microstructures have been ana-

lyzed and it is observed that the initial yield stress of each slip system follows a Hall-Petch trend, which is more faithfully captured when displacement fields at the microstructural level are considered. Three interaction parameters could also be identified. Their values varied significantly with respect to their initial guesses.

The present results show that crystal plasticity parameters can be identified at the grain level. However, they are more challenging since a lot of information is missing (e.g., underlying microstructure, average stress in the region of interest). If more complex loading histories are considered, it is likely that more parameters could be identified (in particular those associated with kinematic hardening). To check such hypotheses, the sensitivity analysis used herein can also be considered. Other crystal plasticity laws may also be tested. 
One key information that was missing is associated with the true $3 \mathrm{D}$ microstructure. In this work, 2D assumptions have been investigated to address this question. However, various approaches may be considered in future studies. First, diffraction contrast tomography allows this type of information to be retrieved in a non destructive way [23]. It may be combined with global digital volume correlation [24] to compare measured 3D displacement fields with those simulated numerically. It is worth noting that the $3 \mathrm{D}$ simulations of such complex microstructures require an extensive computation power, which is even more critical when updating procedures are to be followed. Second, columnar microstructures may be tailored so that the surface information is representative of the whole $3 \mathrm{D}$ polycrystal $[15,16,25]$. Although difficult to reach for industrial materials (such as martensitic or bainitic steels, fine grain metals), this type of situation will remain more tractable with the tools developed herein.

\section{Acknowledgements}

The authors acknowledge the financial support of EDF within R\&D LOCO and PERFORM60 (www.perform60.net) projects. François Curtit, Ghiath Monnet, Jean-Michel Proix and Nicolas Rupin are thanked for fruitful discussions. 


\section{AppendixA. Crystal plasticity constitutive relationships and parameters}

At a single crystal level, the resolved shear stress $\tau_{s}$ is determined on each slip system $s$ from the stress tensor $\sigma$

$$
\tau_{s}=\sigma: \mu_{s}
$$

where $\boldsymbol{\mu}_{s}$ is Schmid's tensor also known as orientation tensor. The crystal plasticity model chosen in this study has been proposed by Méric et al. [17]. The plastic flow relationship expressed for each slip systems $s$ provides the shear strain rate $\gamma_{s}$ as a function of $\tau_{s}$

$$
\dot{\gamma}_{s}=\dot{p}_{s} \frac{\tau_{s}-c \alpha_{s}}{\left|\tau_{s}-c \alpha_{s}\right|}
$$

and

$$
\dot{p}_{s}=\left\langle\frac{\left|\tau_{s}-c \alpha_{s}\right|-r_{s}\left(p_{s}\right)}{k}\right\rangle_{+}^{n}
$$

where $c$ is a kinematic hardening modulus, and $k$ and $n$ are viscosity coefficients. The brackets $\langle.\rangle_{+}$denote the positive part of their argument. The crystal plasticity law has two hardening contributions, namely, one relationship associated with isotropic hardening $r_{s}$

$$
r_{s}=r_{0}+q\left(\sum_{r=1}^{12} h_{s r}\left(1-e^{-b p_{r}}\right)\right)
$$

and another one associated with kinematic hardening $\alpha_{s}$

$$
\dot{\alpha}_{s}=\dot{\gamma}_{s}-d \alpha_{s} \dot{p}_{s}
$$

where $r_{0}, q, b$ and $d$ are constitutive parameters, and $h_{s r}$ the coefficients of the interaction matrix between slip systems detailed in Table A.4. Last, the viscoplastic strain rate $\dot{\epsilon}_{p}$ is assumed to be the sum of plastic glide along all the slip systems

$$
\dot{\boldsymbol{\epsilon}}_{p}=\sum_{s=1}^{12} \dot{\gamma}_{s} \boldsymbol{\mu}_{s}
$$

For each specimen, the parameters associated with isotropic hardening have been identified by homogenization from the experimental curve with Berveiller-Zaoui's model [19]. Their values 
are gathered in Table A.5. To model the anisotropic elastic behavior of the material, cubic symmetry is assumed. The cubic elastic constants $C_{1111}, C_{1122}$ and $C_{1212}$ used are those obtained by acoustic measurements [26] and are gathered in Table A.6. The parameters of the crystal plasticity law also given in Table A.6 are those identified for the same 316LN plate, with the identification method proposed in Ref. [27]. All these values are considered as references in this study.

Table A.4: Interaction matrix between slip systems

\begin{tabular}{|l|l|l|l|l|l|l|l|l|l|l|l|l|}
\hline 1 & 2 & 3 & 4 & 5 & 6 & 7 & 8 & 9 & 10 & 11 & 12 \\
\hline 1 & $h_{1}$ & $h_{2}$ & $h_{2}$ & $h_{4}$ & $h_{5}$ & $h_{5}$ & $h_{5}$ & $h_{6}$ & $h_{3}$ & $h_{5}$ & $h_{3}$ & $h_{6}$ \\
\hline 2 \\
\hline 3
\end{tabular}


Table A.5: Values of the parameters associated with isotropic hardening of the crystal plasticity law [17] identified by homogenization for each specimen

\begin{tabular}{|c|c|c|c|}
\hline Specimen & $r_{0}(\mathrm{MPa})$ & $q(\mathrm{MPa})$ & $b(-)$ \\
\hline$A_{70}$ & 50 & 163 & 1.2 \\
\hline$B_{10}$ & 69 & 183 & 1.2 \\
\hline$C_{10}$ & 60 & 195 & 1.2 \\
\hline$D_{50}$ & 41 & 179 & 1.2 \\
\hline$E_{1000+}$ & 29 & 159 & 0.4 \\
\hline
\end{tabular}

Table A.6: Values of the parameters of cubic elasticity and of the crystal plasticity law [17] kept constant in this study

\begin{tabular}{|c|c|c|c|c|c|c|c|c|}
\hline$C_{1111}(\mathrm{GPa})$ & \multicolumn{2}{|c|}{$C_{1122}(\mathrm{GPa})$} & $C_{1212}(\mathrm{GPa})$ & $n(-)$ & \multicolumn{2}{|c|}{$k\left(\mathrm{MPa} . \mathrm{s}^{1 / n}\right)$} & $c(\mathrm{GPa})$ & $d(-)$ \\
\hline 207 & \multicolumn{2}{|l|}{133} & 117 & 10 & \multicolumn{2}{|c|}{25} & 10.4 & 340 \\
\hline & $h_{1}(-)$ & $h_{2}(-)$ & $h_{3}(-)$ & $h_{4}(-)$ & $h_{5}(-)$ & $h_{6}(-)$ & & \\
\hline & 1.0 & 1.0 & 0.6 & 12.3 & 1.6 & 1.3 & & \\
\hline
\end{tabular}




\section{References}

[1] F. Roters, P. Eisenlohr, L. Hantcherli, D.D. Tjahjanto, T.R. Bieler, and D. Raabe, 2010. Overview of constitutive laws, kinematics, homogenization and multiscale methods in crystal plasticity finite-element modeling: Theory, experiments, applications. Acta Materialia. $58,1152-1211$.

[2] R.A. Lebensohn, A.D. Rollett, P. Suquet, 2011. Fast Fourier transform-based modeling for the determination of micromechanical fields in polycrystals. Journal of Materials. 63, $13-18$.

[3] A. Guery, F. Hild, F. Latourte, S. Roux, 2016. Slip activities in polycrystals determined by coupling DIC measurements with crystal plasticity calculations. International Journal of Plasticity. 81, 249-266.

[4] M.H.H. Meuwissen, C.W.J. Oomens, F.P.T. Baaijens, R. Peterson, J.D. Janssen, 1998. Determination of the elasto-plastic properties of aluminium using a mixed numericalexperimental method. Journal of Materials Processing Technology. 75, 204-211.

[5] J. Kajberg, K. Sundin, L. Melin, P. Ståhle, 2004. High strain rate tensile testing and viscoplastic parameter identification using microscopic high-speed photography. International Journal of Plasticity. 20, 561-575.

[6] F. Latourte, A. Chrysochoos, S. Pagano, B. Wattrisse, 2008. Elastoplastic Behavior Identification for Heterogeneous Loadings and Materials. Experimental Mechanics. 48, 435-449.

[7] F. Mathieu, H. Leclerc, F. Hild, S. Roux, 2015. Estimation of elastoplastic parameters via weighted FEMU and integrated-DIC. Experimental Mechanics. 55, 105-119.

[8] R. Gras, H. Leclerc, F. Hild, S. Roux, J. Schneider, 2015. Identification of a set of macroscopic elastic parameters in a 3D woven composite: Uncertainty analysis and regularization. International Journal of Solids and Structures. 55, 2-16. 
[9] E. Héripré, M. Dexet, J. Crépin, L. Gélébart, A. Roos, M. Bornert, D. Caldemaison, 2007. Coupling between experimental measurements and polycrystal finite element calculations for micromechanical study of metallic materials. International Journal of Plasticity. 23, $1512-1539$.

[10] H. Lim, J.D. Carroll, C.C. Battaile, B.L. Boyce, C.R. Weinberger, 2015. Quantitative comparison between experimental measurements and CP-FEM predictions of plastic deformation in a tantalum oligocrystal. International Journal of Mechanical Sciences. 92, 98-108.

[11] M.A. Sutton, N. Li, D.C. Joy, A.P. Reynolds, X. Li, 2007. Scanning electron microscopy for quantitative small and large deformation measurements Part I: Sem imaging at magnifications from 200 to 10.000. Experimental Mechanics. 47, 775-787.

[12] M.A. Sutton, N. Li, D. Garcia, N. Cornille, J.-J. Orteu, S.R. McNeill, H.W. Schreier, X. Li, A.P. Reynolds, 2007. Scanning electron microscopy for quantitative small and large deformation measurements Part II: Experimental validation for magnifications from 200 to 10.000. Experimental Mechanics. 47, 789-804.

[13] T. Hoc, J. Crépin, L. Gélébart, A. Zaoui, 2003. A procedure for identifying the plastic behavior of single crystals from the local response of polycrystals. Acta Materialia. 51, $5477-5488$.

[14] C.C. Tasan, J.P.M., Hoefnagels, M. Diehl, D. Yan, F. Roters, D. Raabe, 2014. Strain localization and damage in dual phase steels investigated by coupled in-situ deformation experiments and crystal plasticity simulations. International Journal of Plasticity. 63, 198-210.

[15] H. Lim, J.D. Carroll, C.C. Battaile, T.E. Buchheit, B.L. Boyce, C.R. Weinberger, 2014. Grain-scale experimental validation of crystal plasticity finite element simulations of tantalum oligocrystals. International Journal of Plasticity. 60, 1-18.

[16] M. Montagnat, O. Castelnau, P.D. Bons, S.H. Faria, O. Gagliardini, F. Gillet-Chaulet, F. Grennerat, A. Griera, R.A Lebensohn, H. Moulinec, J. Roessiger, P. Suquet, 2014. Multiscale modeling of ice deformation behavior. Journal of Structural Geology. 61, 78-108. 
[17] L. Méric, P. Poubanne, G. Cailletaud, 1991. Single crystal modeling for structural calculations: Part 1 - Model Presentation. Journal of Engineering Materials and Technology. 113, $162-170$.

[18] A. Zeghadi, F. N'Guyen, S. Forest, A.-F. Gourgues, O. Bouaziz, 2007. Ensemble averaging stress-strain fields in polycrystalline aggregates with a constrained surface microstructure Part 1: Anisotropic elastic behaviour. Philosophical Magazine. 87, 1401-1424.

[19] M. Berveiller, A. Zaoui, 1979. An extension of the self-consistent scheme to plastically flowing polycrystal. Journal of the Mechanics and Physics of Solids. 26, 325-344.

[20] F. Hild and S. Roux, 2012. Comparison of local and global approaches to digital image correlation. Experimental Mechanics, 52:1503-1519.

[21] F. Barbe, S. Forest, G. Cailletaud, 2001. Intergranular and intragranular behavior of polycrystalline aggregates. Part 2: Results. International Journal of Plasticity. 17, 537-563.

[22] B. Devincre, L. Kubin, T. Hoc, 2006. Physical analyses of crystal plasticity by DD simulations. Scripta Materialia. 54, 741-746.

[23] W. Ludwig, S. Schmidt, E.M. Lauridsen, H.F. Poulsen, 2008. X-ray diffraction contrast tomography: a novel technique for three-dimensional grain mapping of polycrystals. I. Direct beam case. Journal of Applied Crystallography. 41, 302-309.

[24] S. Roux, F. Hild, P. Viot, D. Bernard, 2008. Three dimensional image correlation from X-Ray computed tomography of solid foam. Composites: Part A 39, 1253-1265.

[25] J.D. Carroll, B.G. Clark, T.E. Buchheit, B.L. Boyce, C.R. Weinberger, 2013. An experimental statistical analysis of stress projection factors in BCC tantalum. Materials Science and Engineering: A. 581, 108-118.

[26] H. Ledbetter, 1987. Monocrystal elastic constants in the ultrasonic study of welds. Ultrasonics. 23, 9-13. 
[27] Y. Guilhem, 2011. Etude numérique des champs mécaniques locaux dans les agrégats polycristallins d'acier 316L sous chargement de fatigue. PhD thesis, Mines ParisTech (in French). 